\title{
Convergence in the no-core shell model with low-momentum two-nucleon interactions
}

\author{
S.K. Bogner ${ }^{a, b}$, R.J. Furnstahl ${ }^{\mathrm{a}, *}$, P. Maris ${ }^{\mathrm{c}}$, R.J. Perry ${ }^{\mathrm{a}}$, \\ A. Schwenk ${ }^{\mathrm{d}}$, J.P. Vary ${ }^{\mathrm{c}}$ \\ ${ }^{a}$ Department of Physics, The Ohio State University, Columbus, OH 43210 \\ ${ }^{\mathrm{b}}$ National Superconducting Cyclotron Laboratory and Department of Physics and \\ Astronomy, Michigan State University, East Lansing, MI 48844 \\ ${ }^{\mathrm{c}}$ Department of Physics and Astronomy, Iowa State University, Ames, IA 50011 \\ d TRIUMF, 4004 Wesbrook Mall, Vancouver, BC, Canada, V6T $2 A 3$
}

\begin{abstract}
The convergence of no-core shell model (NCSM) calculations using renormalization group evolved low-momentum two-nucleon interactions is studied for light nuclei up to ${ }^{7} \mathrm{Li}$. Because no additional transformation was used in applying the NCSM framework, the energy calculations satisfy the variational principle for a given Hamiltonian. Dramatic improvements in convergence are found as the cutoffs are lowered. The renormalization group equations are truncated at two-body interactions, so the evolution is only approximately unitary and converged energies for $A \geqslant 3$ vary with the cutoff. This approximation is systematic, however, and for useful cutoff ranges the energy variation is comparable to natural-size truncation errors inherent from the initial chiral effective field theory potential.
\end{abstract}

\section{Introduction}

The nonperturbative nature of inter-nucleon interactions is strongly scale or resolution dependent and can be radically altered by using renormalization group (RG) methods to decouple low- and high-momentum modes in nuclear Hamiltonians $[1,2,3,4,5,6,7$, . A consequence of this decoupling is that

\footnotetext{
* Corresponding author.

Email addresses: bogner@nscl.msu.edu (S.K. Bogner), furnstahl.1@osu.edu (R.J. Furnstahl), pmaris@iastate.edu (P. Maris), perry@mps.ohio-state.edu

(R.J. Perry), schwenk@triumf.ca (A. Schwenk), jvary@iastate.edu (J.P. Vary).
} 
few- and many-body calculations become more tractable at lower resolutions [8, 9, 11, 12, which implies that ab-initio basis expansion methods such as the No-Core Shell Model (NCSM) should exhibit much improved convergence properties. Moreover, the RG approach has the advantage of being able to vary the cutoff as a tool to probe the quality of the many-body approximation and to provide estimates of the size of omitted terms in the Hamiltonian (e.g., three-nucleon interactions) [8].

In this paper, we present NCSM calculations of ground states and some lowlying natural-parity excited states for light nuclei $\left({ }^{3} \mathrm{H},{ }^{4} \mathrm{He},{ }^{6} \mathrm{He},{ }^{6} \mathrm{Li}\right.$ and $\left.{ }^{7} \mathrm{Li}\right)$ using RG-evolved nucleon-nucleon (NN) interactions as input. These potentials include similarity renormalization group (SRG) interactions and both sharp [3] and smooth [5] cutoff versions of low-momentum interactions $V_{\text {low }} k$ derived from one of the $\mathrm{N}^{3} \mathrm{LO}$ effective field theory (EFT) two-nucleon potentials [1314]. The dependence of convergence properties on the cutoff is of particular interest 1 In applying the NCSM framework, no additional transformation of the Hamiltonian is performed. Thus, the energy calculations here satisfy the variational principle for the NN interaction at each cutoff. Details of the NCSM approach can be found in Refs. [15,16,17,18,19] and previous applications of the NCSM to soft bare NN potentials are described in Refs. [20,21,22].

All of the calculations are for NN interactions only. Since the physics is qualitatively incomplete without including at least three-nucleon (3N) interactions, these should be viewed as baseline calculations that set the stage for inclusion of $3 \mathrm{~N}$ interactions in the near future [23]; some areas of investigation have been deferred until then. The additional computational costs of $3 \mathrm{~N}$ interactions will severely limit the feasible basis sizes for all but the lightest nuclei. So rather than use large spaces to ensure convergence of all the NN-only calculations, we have used relatively small spaces for all nuclei. With the improved convergence rates at lower momentum cutoffs, we have been able to perform all of the calculations on a small computer cluster. This is sufficient to establish that the scaling of computer resources should be favorable for adding three-body interactions.

The RG equations inevitably shift strength from two-body to many-body interactions as $\lambda$ or $\Lambda$ is lowered, even if many-body interactions are initially zero. If we only keep the NN part, the transformations are only approximately unitary and few-body binding energies will change ("run") with $\lambda$ or $\Lambda$. This approximation is not a problem because the initial chiral EFT potentials have truncation errors and associated theoretical error bands for observables. We

1 The SRG potentials are characterized by a flow parameter $\lambda$ that sets the scale of decoupling of low-energy and high-energy physics, thus playing the role of a cutoff. For convenience we will refer to both the SRG $\lambda$ and the $V_{\text {low } k} \Lambda$ as cutoffs. 
can ensure that the variation of energies (and other observables) are comparable to the EFT truncation errors and that the hierarchy of many-body forces is preserved by restricting the range of RG cutoffs. By tracking the running of the energies, we can assess the expected net contributions from many-body forces in a more complete future calculation.

We stress at the outset that although the converged (or extrapolated) binding energies for certain cutoffs are quite close to experimental binding energies, this should not be taken as motivation for using those cutoffs and neglecting three-body interactions. Since nuclear matter calculated with low-momentum interactions does not saturate with NN only [4], it is clear that the importance of $3 \mathrm{~N}$ contributions will increase as one moves higher in the table of nuclides.

The outline of the paper is as follows. In Section 2 we look at the convergence of ground-state energies of light nuclei in a harmonic-oscillator basis using SRG-evolved momentum-space interactions. We find that decoupling documented for two-body systems in Ref. [24] is also present in heavier nuclei (with NN only). The SRG convergence patterns are compared to those for $V_{\text {low } k}$ potentials using regulators with varying degrees of smoothness in Section 3. A simple extrapolation procedure, which is needed for the larger cutoffs in nuclei with $A>3$ and for all cutoffs in ${ }^{7} \mathrm{Li}$, yields energies with error bars that exhibit the net evolution ("running") of three-body (and higher-body) contributions. The error bars reflect the NCSM convergence and the cutoff variation assesses the impact of missing many-body interactions. These results are exhibited in Section 4. Convergence for selected excited states and radii are shown in Section 5. We conclude in Section 6 with a summary and outlook for future calculations.

\section{Convergence of SRG Ground-State Energies}

In this section, we present results for ground-state energies of light nuclei for a range of harmonic-oscillator basis sizes and oscillator parameters using potentials evolved with the similarity renormalization group [25,26,27]. The SRG flow is a series of unitary transformations parametrized by a flow variable $s$ :

$$
H_{s}=U(s) H U^{\dagger}(s) \equiv T+V_{s},
$$

where $H$ is the original Hamiltonian (corresponding to $s=0$ ) and $T$ is the kinetic energy, which is taken to be independent of $s$ (thereby defining $V_{s}$ ). The choice of transformations leads to the flow equation for $V_{s}$,

$$
\frac{d V_{s}}{d s}=\left[\left[T_{D}, H_{s}\right], H_{s}\right]=\left[\left[T_{D}, V_{s}\right], H_{s}\right]
$$


which is evaluated here for NN interactions in a partial-wave momentum basis [6,24,28]. The operator $T_{D}$ is a diagonal matrix in this basis. The results of Refs. [6,24] and all results here use $T_{D}=T$, but we have also checked convergence for $T_{D}=T^{2}$ and $T_{D}=T^{3}$. For $T_{D}=T$ in momentum representation, $\lambda \equiv 1 / s^{1 / 4}$ is a more useful flow variable that can be thought of as a cutoff on momentum transfers, and we use it exclusively from now on.

We use primarily the $\mathrm{N}^{3} \mathrm{LO}$ potential from Ref. [13], which has been the subject of previous investigations in the NCSM [18,19]. In those investigations, Lee-Suzuki transformations were used to derive effective interactions that improved convergence, but which lose the variational property when making a cluster approximation. In contrast, the present calculations use interactions evolved in free space, so they simply represent alternative Hamiltonians; thus the calculations retain the variational principle. Because the evolution to lower momenta yields nearly universal interactions for $\lambda \lesssim 2 \mathrm{fm}^{-1}[1,3,56$, convergence properties starting from other initial $\mathrm{N}^{3} \mathrm{LO}$ [14] or phenomenological potentials [29] are quantitatively similar. For simplicity, in these first NCSM calculations we evolve only the neutron-proton part of nuclear forces, with Coulomb added after the RG evolution.

The choice of $T_{D}=T$ in Eq. (2) leads to a partial diagonalization of the potentials in momentum representation, which is the source of decoupling of low- and high-momentum contributions. We expect reduced short-range correlations and improved convergence in almost any basis as a result of this decoupling. The evolution modifies only the short-distance part of operators; for the Hamiltonian this is important to maintain the hierarchy of many-body forces in the initial EFT Hamiltonian (as long as we don't evolve too far). However, one could also consider tailoring the evolution to a particular basis such as harmonic oscillators. In this case, choosing $T_{D}$ to be the one-body harmonic oscillator Hamiltonian (with a two-body term added to the potential as usual with the NCSM [15,16,17]) will yield a flow to more diagonal and therefore more decoupled potentials in this basis, accelerating convergence. The downside is that there may be serious negative consequences for longdistance operators and many-body forces, analogous to what is observed for Lee-Suzuki transformations in the NCSM [30]. These issues will be explored in a future investigation.

Matrix elements of the momentum-space potential are evaluated in a harmonicoscillator basis for input to the Many-Fermion Dynamics (MFD) code [31], which constructs the many-body matrix elements and performs the diagonalization. The basis is specified by the oscillator parameter $\hbar \Omega$ and by $N_{\max }$. $N_{\text {max }}$ defines the maximum number of oscillator quanta (increments based on sums of single particle quanta, $2 n+l$ ) allowed above the lowest many-body configuration for a given nucleus. Thus $N_{\max }=2$ implies the inclusion of single-particle excitations up to two major oscillator shells or two particles 
excited simultaneously by one major shell above the lowest-energy oscillator configuration.

We view the present NN-only calculations as setting the baseline for upcoming calculations that will include $3 \mathrm{~N}$ forces. Because the latter will have significant computational costs that will limit the maximum basis size that can be used, we study convergence for different $\lambda$ values with at most $N_{\max }=12$ and dimension sizes of order $10^{7}$ or less. This enables a significant number of exploratory cases to be easily and quickly examined on a small computer cluster, including the evaluation of a suite of experimental observables for each case. The basis dimension for ${ }^{2} \mathrm{H}$ ranges from 24 for $N_{\max }=2$ to 4200 for $N_{\max }=12$, for ${ }^{6} \mathrm{Li}$ from 800 for $N_{\max }=2$ to $9,692,634$ for $N_{\max }=10$ and for ${ }^{7} \mathrm{Li}$ from 1961 for $N_{\max }=2$ to $6,150,449$ for $N_{\max }=8$. Extending these calculations to more powerful computers for including $3 \mathrm{~N}$ forces is straightforward.

In Figs. 1 through 6, the ground-state energies of ${ }^{2} \mathrm{H},{ }^{3} \mathrm{H},{ }^{4} \mathrm{He},{ }^{6} \mathrm{He},{ }^{6} \mathrm{Li}$, and ${ }^{7} \mathrm{Li}$ are plotted against the harmonic-oscillator parameter $\hbar \Omega$ for a range of $N_{\text {max }}$ values. The figures with $A \leqslant 4$ show results for the initial chiral $\mathrm{N}^{3} \mathrm{LO}$ potential and three SRG-evolved potentials with $\lambda=3.0,2.0$, and $1.5 \mathrm{fm}^{-1}$. For $A=6$ and $A=7$, our largest spaces were very far from convergence with the initial potential, so we show only SRG-evolved potentials with $\lambda=3.0$, $2.0,1.5$, and $1.0 \mathrm{fm}^{-1}$.

It may appear in Fig. 1 that the deuteron is converged to different groundstate energies for different $\lambda$ values. However, the results simply reflect the slow convergence of the weakly bound deuteron in a harmonic oscillator basis; all converge with sufficiently large spaces to the same energy as the unevolved potential. Only for $\lambda=1.5 \mathrm{fm}^{-1}$ is the energy converged at the $10 \mathrm{keV}$ level by $N_{\max }=12$. (For the other cutoffs, there is an irregular convergence trend for which $N_{\max }=12$ is very close to $N_{\max }=10$, but then $N_{\max }=14$ will be lowered further.) The convergence rate for ${ }^{3} \mathrm{H}$ in Fig. 2 is similar when viewed on the same scale. In contrast to the deuteron, the convergence is to noticeably different energies in large spaces (indicated by the dotted lines) because the SRG evolution truncated to NN-only is only approximately unitary for $A \geqslant 3$. The spread of energies is a measure of this approximation; for the range of $\lambda$ 's shown, it is the same order as the truncation error from omitting $3 \mathrm{~N}$ forces in the original Hamiltonian. The running of energies and its implications are discussed further in Section 4 .

In all cases we find rapidly improving convergence with lowered $\lambda$ down to $\lambda=1.0 \mathrm{fm}^{-1}$, which is consistent with an expected decrease in correlations in ground-state wave functions. Power-counting arguments [8] imply that the hierarchy of many-body forces in heavier nuclei could break down by this point (i.e., the $3 \mathrm{~N}$ and higher-body contributions will become comparable to the NN contribution). However, the scaling of many-body forces in the SRG has not 


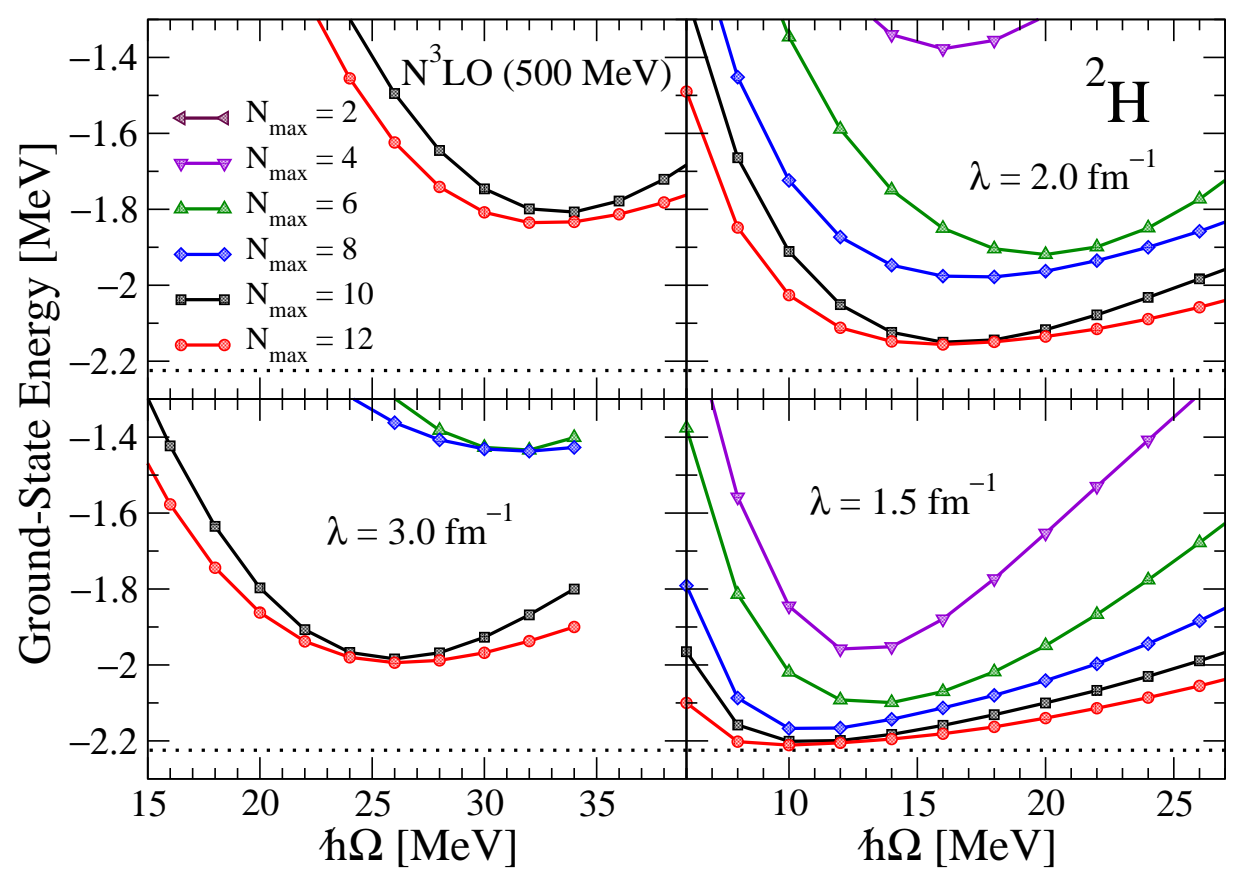

Fig. 1. Ground-state energy of the deuteron as a function of $\hbar \Omega$ at four different values of $\lambda\left(\infty, 3,2,1.5 \mathrm{fm}^{-1}\right)$ for $N_{\max }$ values up to 12 . The initial potential is the $500 \mathrm{MeV} \mathrm{N}^{3} \mathrm{LO}$ NN-only potential from Ref. [13]. The dotted lines show fully converged energies obtained with a sufficiently large basis size.

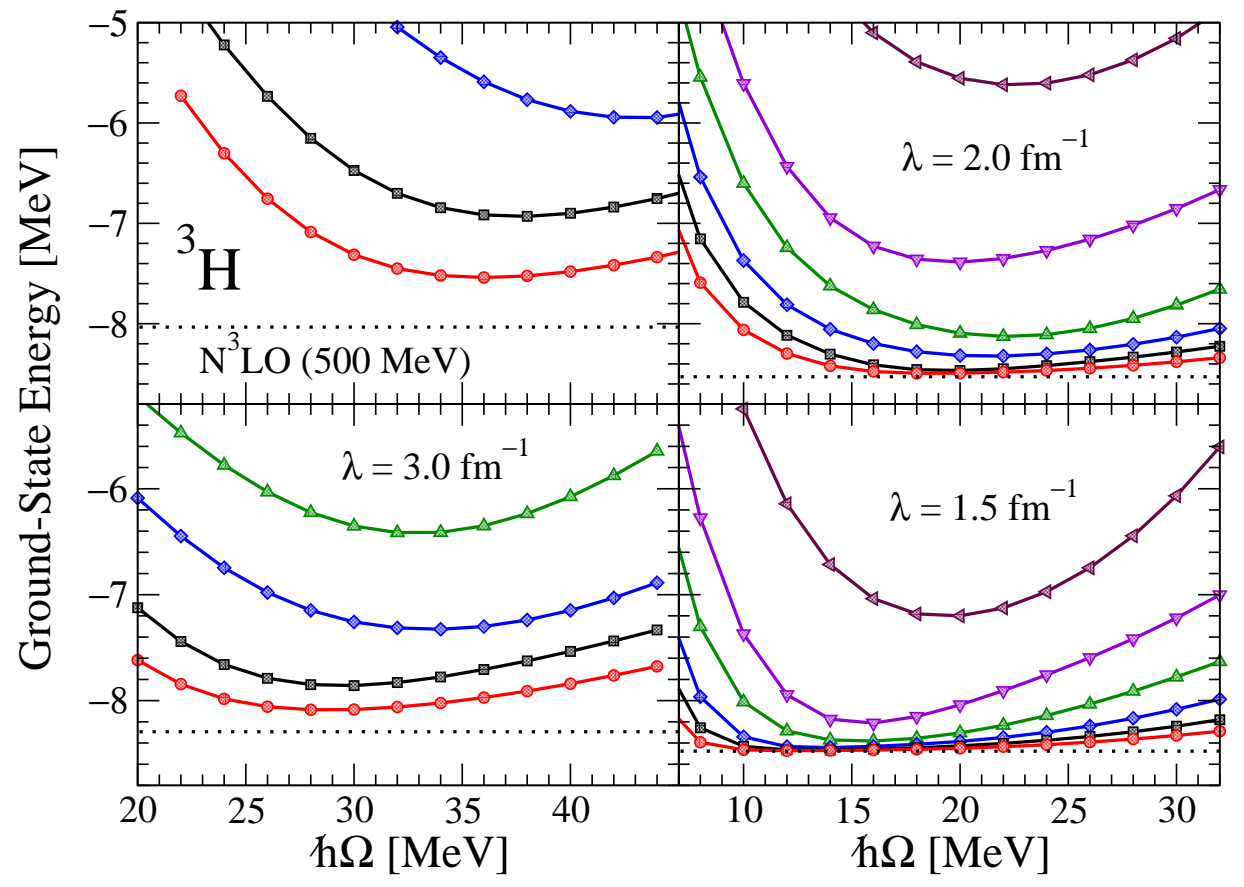

Fig. 2. Ground-state energy of the triton as a function of $\hbar \Omega$ at four different values of $\lambda\left(\infty, 3,2,1.5 \mathrm{fm}^{-1}\right)$. The initial potential is the $500 \mathrm{MeV} \mathrm{N} \mathrm{N}^{3} \mathrm{LO} \mathrm{NN}$-only potential from Ref. [13. The legend from Fig. 1 applies here. The dotted lines show fully converged energies from independent $N_{\max }=48$ calculations using a code from Ref. [32]. 


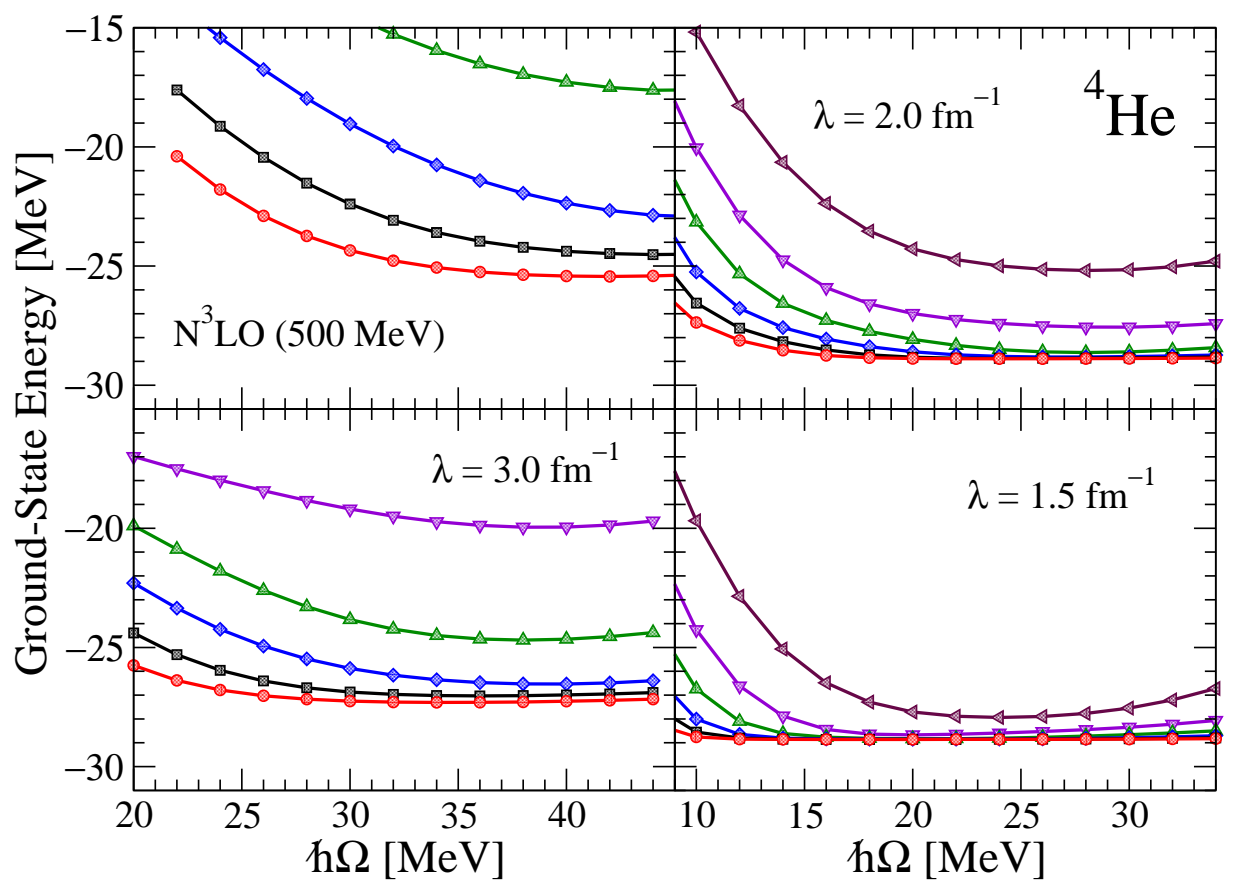

Fig. 3. Ground-state energy of ${ }^{4} \mathrm{He}$ as a function of $\hbar \Omega$ at four different values of $\lambda$ $\left(\infty, 3,2,1.5 \mathrm{fm}^{-1}\right)$. The initial potential is the $500 \mathrm{MeV} \mathrm{N} \mathrm{N}^{3} \mathrm{LO} \mathrm{NN}$-only potential from Ref. [13. The legend from Fig. 1] applies here.

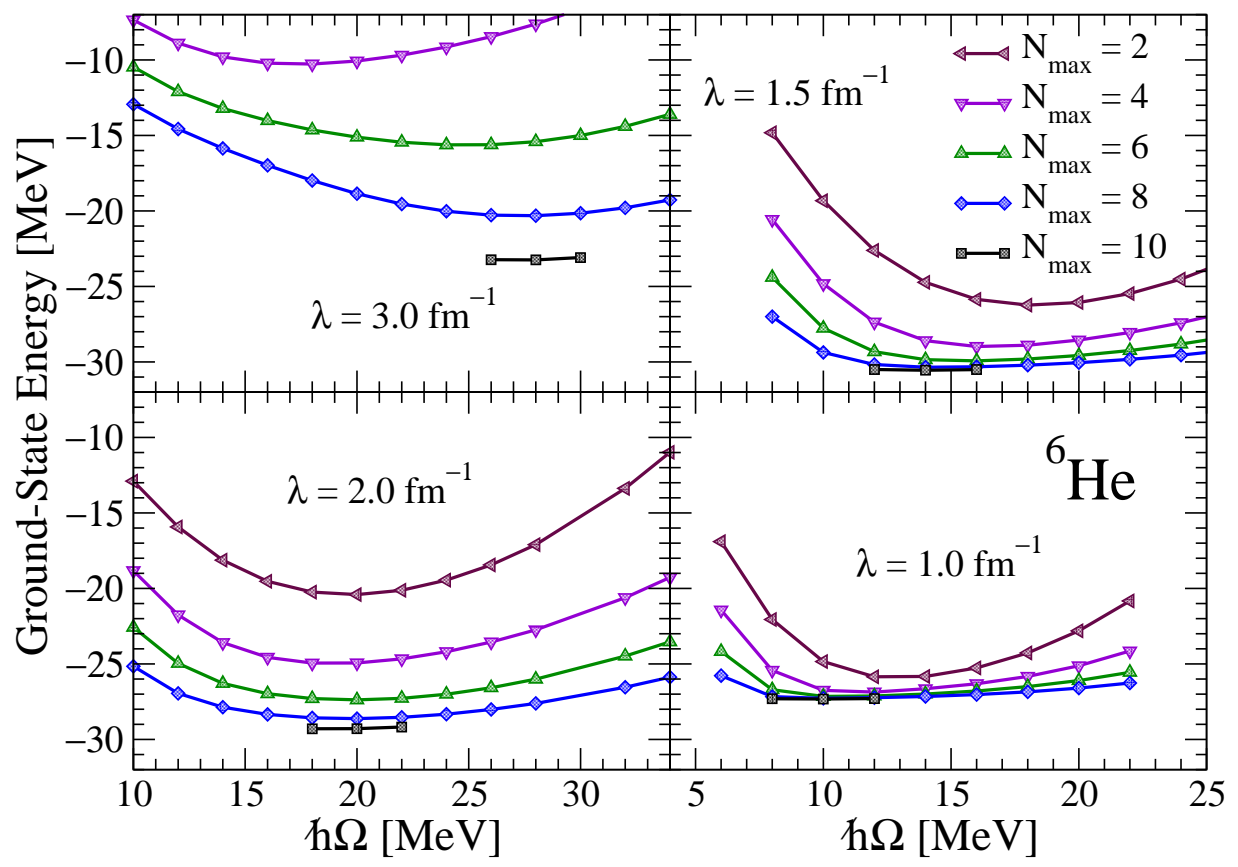

Fig. 4. Ground-state energy of ${ }^{6} \mathrm{He}$ as a function of $\hbar \Omega$ at four different values of $\lambda\left(3,2,1.5,1 \mathrm{fm}^{-1}\right)$. The initial potential is the $500 \mathrm{MeV} \mathrm{N} \mathrm{N}^{3} \mathrm{LO}$ NN-only potential from Ref. [13]. 


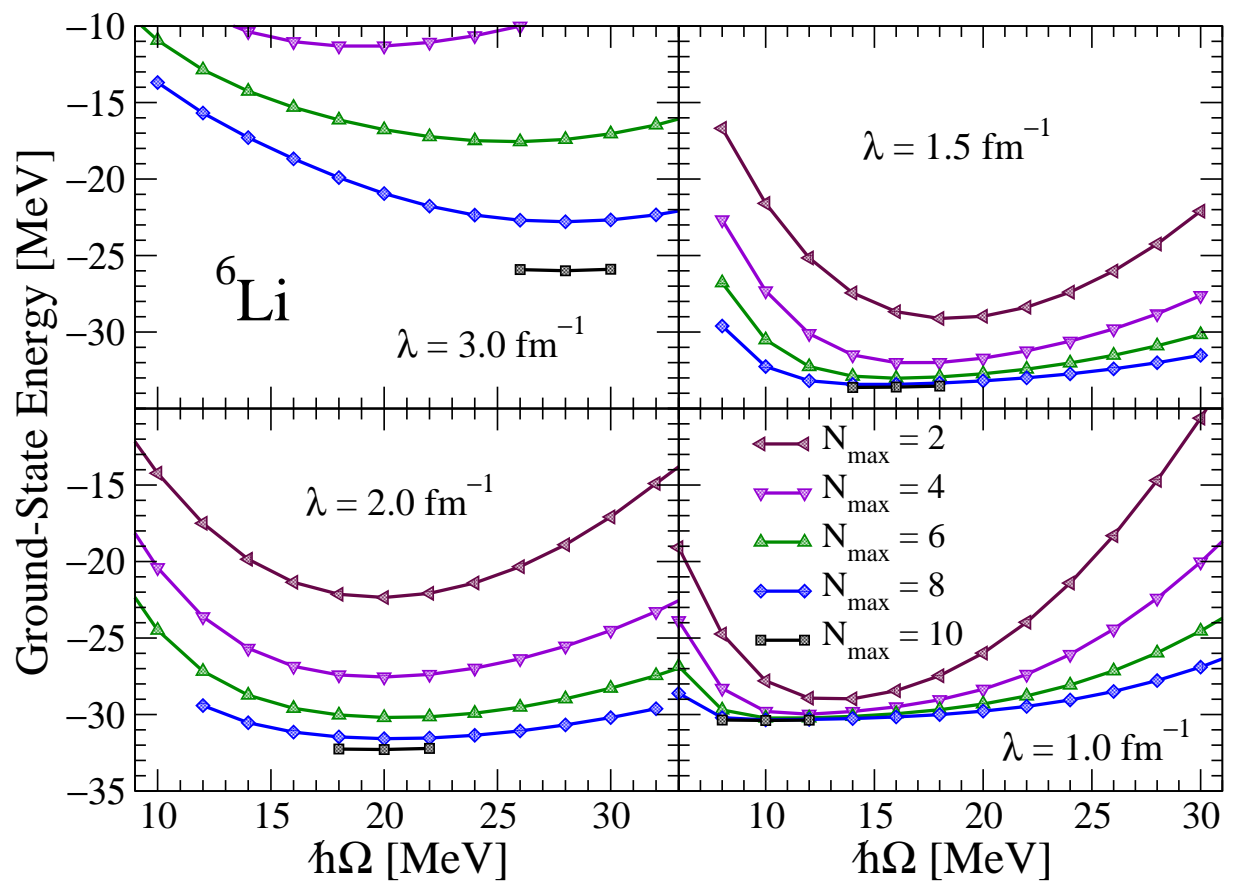

Fig. 5. Ground-state energy of ${ }^{6} \mathrm{Li}$ as a function of $\hbar \Omega$ at four different values of $\lambda\left(3,2,1.5,1 \mathrm{fm}^{-1}\right)$. The initial potential is the $500 \mathrm{MeV} \mathrm{N} \mathrm{N}^{3} \mathrm{LO}$ NN-only potential from Ref. [13].

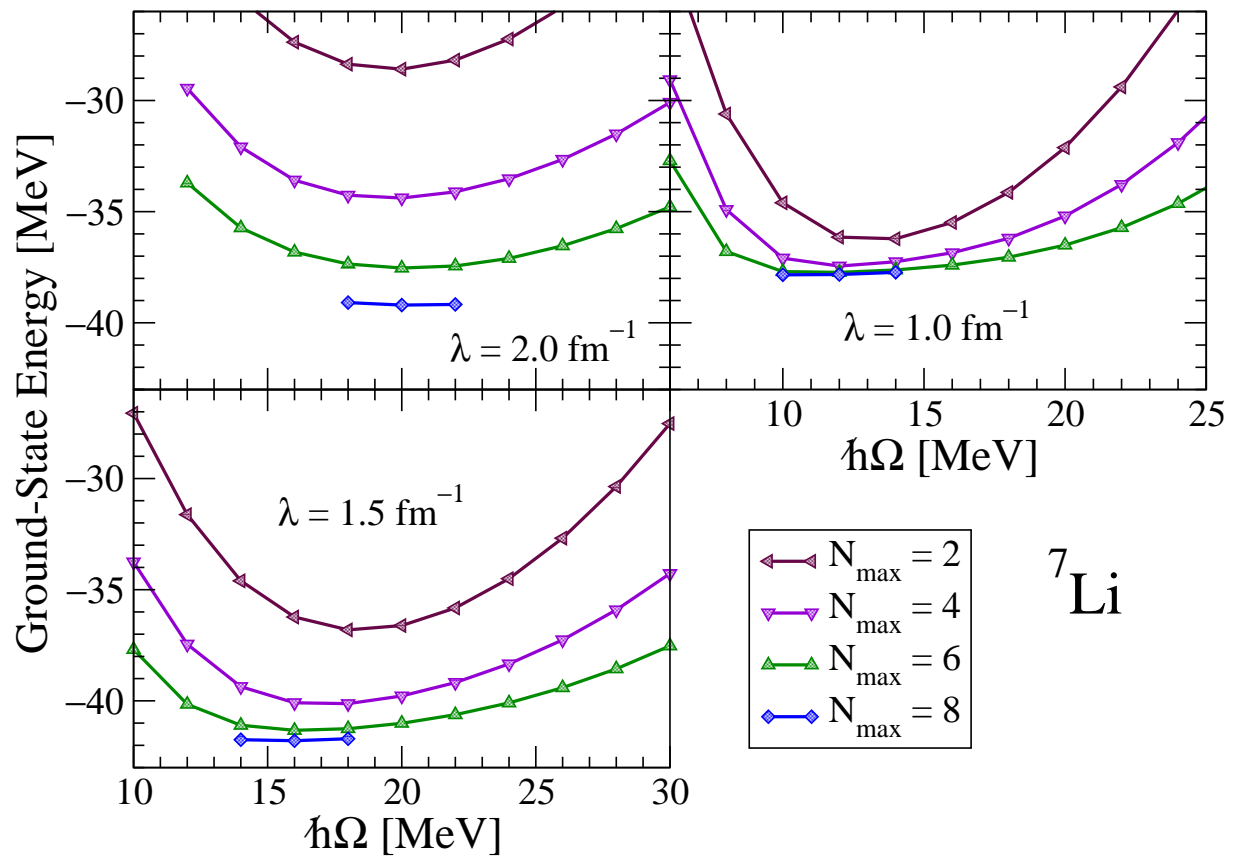

Fig. 6. Ground-state energy of ${ }^{7} \mathrm{Li}$ as a function of $\hbar \Omega$ at three different values of $\lambda$ $\left(2,1.5,1 \mathrm{fm}^{-1}\right)$. The initial potential is the $500 \mathrm{MeV} \mathrm{N}{ }^{3} \mathrm{LO}$ NN-only potential from Ref. [13]. 
yet been established, so the practical lower limit in $\lambda$ for which many-body forces are under control is not yet known. Also, it may be different for light and heavy nuclei. Once we have established the technology to consistently run many-body forces with the SRG (Ref. [23] is a first step in this direction), we will have greater freedom to exploit the choice of $\lambda$ in practical calculations.

In general, the curves of energy vs. $\hbar \Omega$ and the trends with increasing $N_{\max }$ at fixed $\hbar \Omega$ are more systematic with lower $\lambda$. For example, compare ${ }^{6} \mathrm{Li}$ at $\lambda=3.0 \mathrm{fm}^{-1}$ to $\lambda=1.5$ or $2.0 \mathrm{fm}^{-1}$. It is also clear that the larger nuclei have more regular behavior at any $\lambda$. This implies that extrapolations to $N_{\max }=\infty$ will be more robust for heavier nuclei.

The trends with smaller $\lambda$ 's include increasingly reasonable estimates from calculations with smaller $N_{\max }$ values. For example, $N_{\max }=2(4)$ for $\lambda=$ $1.5 \mathrm{fm}^{-1}$ is within $1(0.3) \mathrm{MeV}$ of the converged binding energy for ${ }^{4} \mathrm{He}$ and within 5(1.5) $\mathrm{MeV}$ for ${ }^{6} \mathrm{Li}$. To set the scale for how good these estimates are, we note that the starting $\mathrm{N}^{3} \mathrm{LO}$ potential in ${ }^{4} \mathrm{He}$ is at most bound by $1 \mathrm{MeV}$ for $N_{\max }=2$ and only $8 \mathrm{MeV}$ for $N_{\max }=4$. For $\lambda=3.0 \mathrm{fm}^{-1}, N_{\max }=2(4)$ is still about $14.5(7.3) \mathrm{MeV}$ short of the converged binding energy in ${ }^{4} \mathrm{He}$.

At the same time, with smaller $\lambda$ 's the larger $N_{\max }$ values converge entirely (at the $10 \mathrm{keV}$ level or better) for the lighter nuclei. As $N_{\max }$ increases, the $\hbar \Omega$ dependence gets flatter in a very smooth and systematic way. We also note that for a given $N_{\max }$, the minimum in $\hbar \Omega$ moves toward lower values as $\lambda$ decreases, as is expected for softer interactions. The energies for $\lambda=1.0 \mathrm{fm}^{-1}$ converge remarkably fast. The binding in this case is smaller than for the other $\lambda$ values, which is consistent with the running of the net three-body contribution in Ref. [8] for a sharp $V_{\text {low } k}$ interaction in ${ }^{3} \mathrm{H}$ and ${ }^{4} \mathrm{He}$. Explicit results for the running of the net many-body contribution are given in Section 4 .

We can compare the convergence patterns observed here with those found in previous NCSM investigations. The convergence of Lee-Suzuki effective interaction results with increasing $N_{\max }$ is generally non-monotonic at fixed $\hbar \Omega$. This is seen in numerous examples in the literature (see, e.g., Ref. [22]). It is therefore more challenging to make extrapolations in those cases. However, extrapolations with bare but soft NN interactions, such as JISP16 (Ref. [22]), are more straightforward [33] and similar to the SRG convergence. Extrapolations of SRG results are tested in Section 4 .

In Ref. [24], the SRG was used to demonstrate how running to lower momentum decouples low- and high-momentum contributions to matrix elements of low-energy observables. Only two-body observables were considered. Using the NCSM, we can extend the tests of decoupling with NN interactions to fewbody systems. One test is to apply a cutoff function to the SRG potential, in 
the form

$$
V_{\lambda}\left(k, k^{\prime} ; k_{\max }\right)=e^{-\left(k^{2} / k_{\max }^{2}\right)^{n}} V_{\lambda}\left(k, k^{\prime}\right) e^{-\left(k^{\prime 2} / k_{\max }^{2}\right)^{n}},
$$

where the choice for $n$ controls the smoothness of the cutoff, and then to calculate the ground-state energy as $k_{\max }$ is varied from 0 to $\infty$. This second cutoff (here with $n=8$ ) is simply imposed by hand after the RG cutoff is run as a tool to test decoupling: if there is decoupling of low- and high-momentum contributions, then we should be able to set matrix elements of the potential to zero in a smooth way above some momentum $k_{\max }$ and still get the same answer for low-energy observables. The effect of the second cutoff is shown for the triton in Fig. 7 starting from the $\mathrm{N}^{3} \mathrm{LO}$ potential of Ref. [13. For comparison, we show in Fig. 8 the same calculation but starting with the Argonne $v_{18}$ potential [29].

The convergence with increasing $k_{\max }$ of the binding energy for the bare $(\lambda=$ $\infty)$ interaction to the asymptotic value is determined by the intrinsic cutoffs in the potential and reflects the associated decoupling. Thus for AV18, the energy is converged to good accuracy by $k_{\max } \approx 7 \mathrm{fm}^{-1}$ while for the $\mathrm{N}^{3} \mathrm{LO}$ potential convergence is reached between 4 and $4.5 \mathrm{fm}^{-1}$. (Note that the latter result is much higher than the naive estimate of $2.5 \mathrm{fm}^{-1}$ based on the $500 \mathrm{MeV}$ cutoff, due to a significant high-momentum tail and the associated tensor strength.) As the potential is evolved to lower $\lambda$, the convergence scale for the binding energy is set by $\lambda$. That is, the most rapid changes happen for $k_{\max }$ up to $\lambda$ and then there is a slower approach to the asymptotic value of the energy, which is very well converged in all cases by about $1.4 \lambda$. These details are common for any potential evolved with $T$ to define the SRG [34]. Similar results for other nuclei and a quantitative perturbative analysis of decoupling will be given in Ref. [34].

We have also examined the convergence of the ground-state energy for SRG potentials using $T_{D}=T^{2}$ and $T_{D}=T^{3}$ in Eq. (2) rather than the kinetic energy $T$. The convergence behavior in both cases is almost indistinguishable from those for $T$.

\section{Convergence of $V_{\text {low } k}$ Ground-State Energies}

In this section we compare the convergence for the SRG to that of $V_{\text {low } k}$ interactions with sharp and various smooth regulators. In contrast to the SRG flow equations for $V_{s}$, the $V_{\text {low } k}$ potentials are based on the invariance of the low-energy NN T matrix under changes of a cutoff $\Lambda$ on relative momenta [2,3,5]. This is achieved either through coupled differential equations for the momentum-space matrix elements of $V_{\text {low } k}$ or in integral form through a free-space Lee-Suzuki transformation [1]. The construction and characteristics of $V_{\text {low } k}$ interactions with smooth regulators is described in Ref. [5]. 


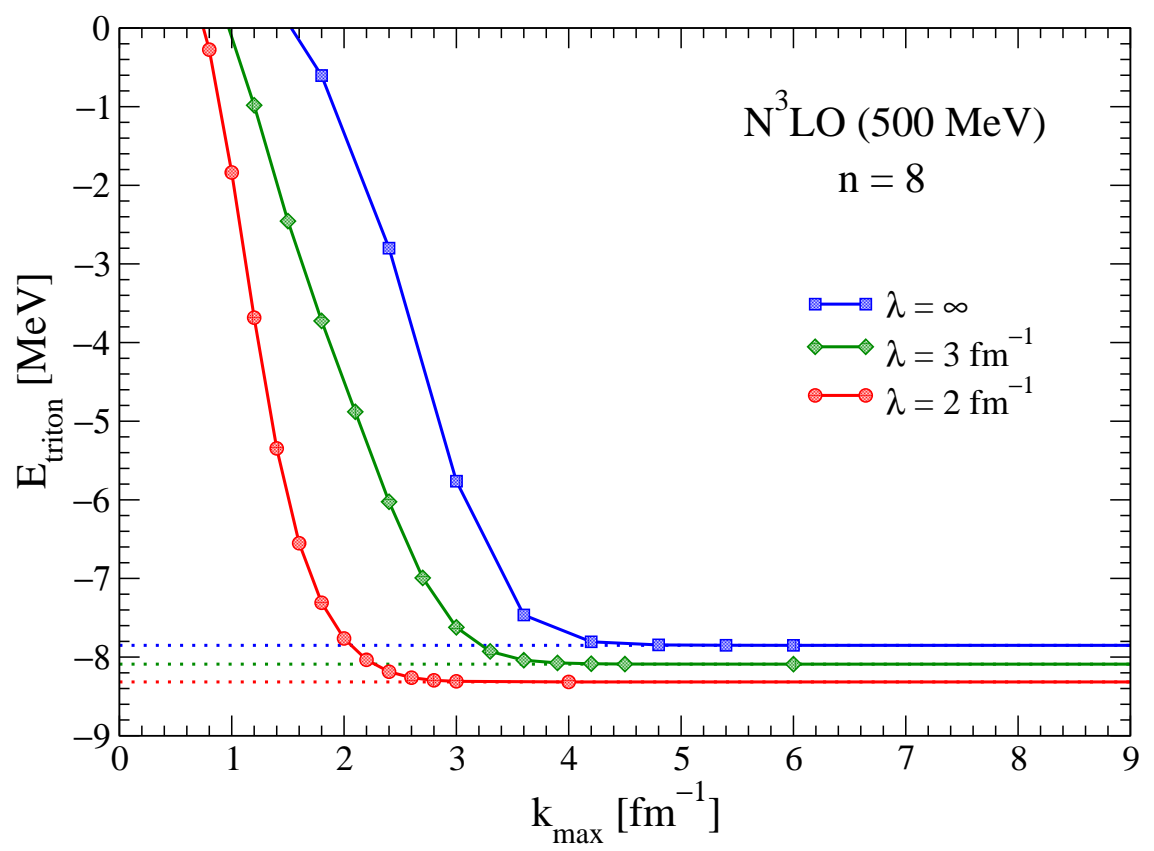

Fig. 7. Decoupling through the SRG. Binding energy as a function of cutoff parameter $k_{\max }$ for three different values of $\lambda$. The cutoff function is $\exp \left[-\left(k^{2} / k_{\max }^{2}\right)^{n}\right]$ with $n=8$. The initial potential is the $500 \mathrm{MeV} \mathrm{N}^{3} \mathrm{LO}$ NN-only potential from Ref. [13]. These results include evolved electromagnetic interactions and charge dependences.

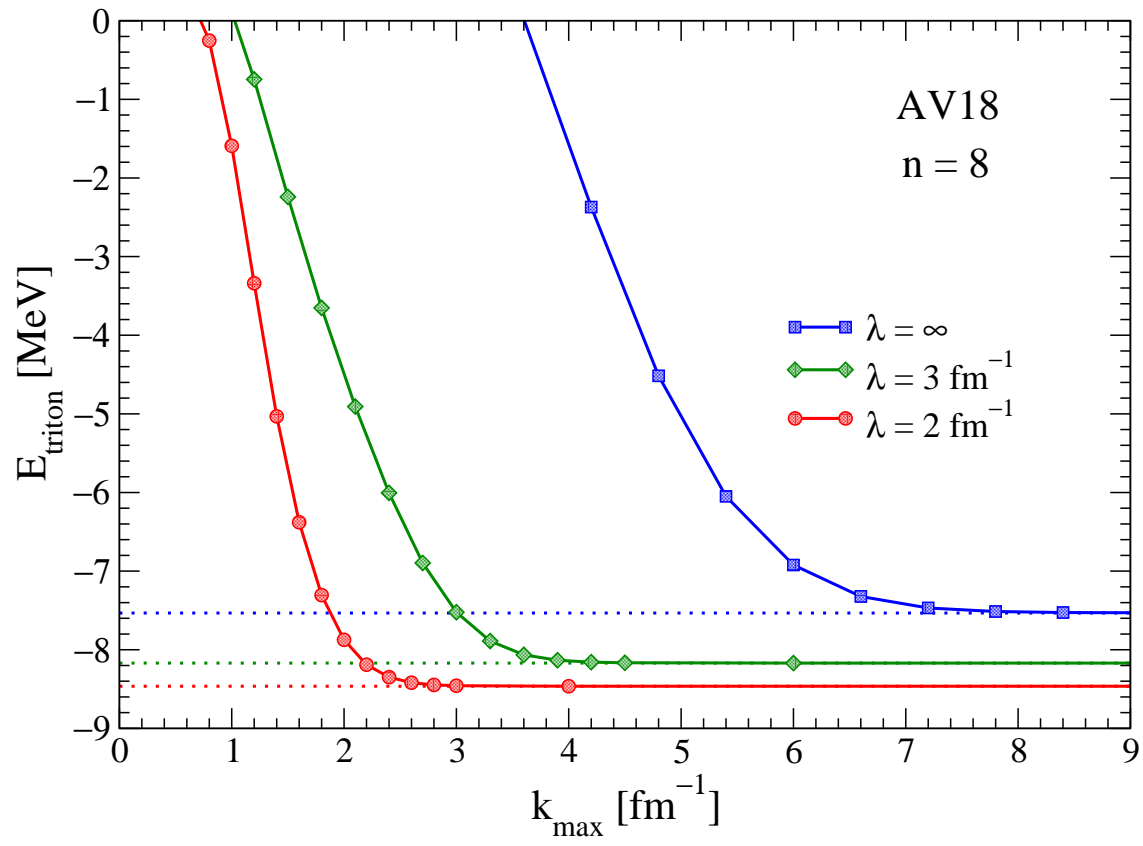

Fig. 8. Decoupling through the SRG. Ground-state energy as a function of cutoff parameter $k_{\max }$ for three different values of $\lambda$. The cutoff function is $\exp \left[-\left(k^{2} / k_{\max }^{2}\right)^{n}\right]$ with $n=8$. The initial potential is NN-only Argonne $v_{18}$ [29]. These results include evolved electromagnetic interactions and charge dependences. 
In previous comparisons between the SRG with flow parameter $\lambda$ and $V_{\text {low }} k$ interactions with momentum cutoff $\Lambda$, the characteristics of the potential and its predictions at low energy were found to be strikingly similar in two-body calculations when $\lambda \approx \Lambda$. The change in convergence with different $\Lambda$ 's is similar to that shown for different $\lambda$ 's in Section 2, Ground-state energies for ${ }^{2} \mathrm{H},{ }^{3} \mathrm{H},{ }^{4} \mathrm{He}$, and ${ }^{6} \mathrm{Li}$ are plotted against the harmonic-oscillator parameter $\hbar \Omega$ for a range of $N_{\max }$ values in Figs. 9 through 12. Each figure shows results for a sharp and two smooth $V_{\text {low } k}$ potentials with $\Lambda=2 \mathrm{fm}^{-1}$ and an SRG potential with $\lambda=2 \mathrm{fm}^{-1}$. The initial potential is the $500 \mathrm{MeV} \mathrm{N} \mathrm{N}^{3} \mathrm{LO}$ potential from Ref. [13].

We find that $V_{\text {low } k}$ potentials with smoother cutoffs (but the same $\Lambda=2 \mathrm{fm}^{-1}$ ) converge more uniformly and that lower $N_{\max }$ estimates are superior. The latter advantage seems to largely disappear as the space gets larger (e.g., see ${ }^{6} \mathrm{Li}$ ). However, extrapolations may be more robust with the SRG and the smoother $V_{\text {low } k}$ potentials. Note that the SRG is a smooth cutoff low-momentum potential. With $T_{D}$ defined with a single power of $T$, its characteristics are closest to those of a $V_{\text {low } k}$ potential with exponential cutoff $\exp \left[-\left(k^{2} / \Lambda^{2}\right)^{4}\right]$ with $\lambda \approx \Lambda$.

The UCOM framework is an alternative approach to using unitary transformations to soften NN potentials and reduce correlations. We can compare the observed SRG convergence to that found for the UCOM potential in Ref. [35], where results for ${ }^{3} \mathrm{H}$ and ${ }^{4} \mathrm{He}$ starting from AV18 are shown in their Fig. 8. For ${ }^{3} \mathrm{H}$ at $N_{\max }=6$ the $\mathrm{UCOM}$ result is about $2 \mathrm{MeV}$ from the converged value and at $N_{\max }=12$ the discrepancy is about $0.75 \mathrm{MeV}$. The SRG calculation with $\lambda=2 \mathrm{fm}^{-1}$ is $0.5 \mathrm{MeV}$ from converged at $N_{\max }=6$ and only $25 \mathrm{keV}$ away at $N_{\max }=12$. With $\lambda=1.5 \mathrm{fm}^{-1}$, the discrepancies are 90 and $4 \mathrm{keV}$, while with $\lambda=3.0 \mathrm{fm}^{-1}$, they are $1.9 \mathrm{MeV}$ and $200 \mathrm{keV}$. If we consider ${ }^{4} \mathrm{He}$, the UCOM result at $N_{\max }=6$ is about $2.5 \mathrm{MeV}$ and at $N_{\max }=10$ about $1 \mathrm{MeV}$ from converged. For the $\mathrm{SRG}$, the $\lambda=1.5 \mathrm{fm}^{-1}$ discrepancies are 30 and $1 \mathrm{keV}$ and at $\lambda=3.0 \mathrm{fm}^{-1}$ they are about 2.6 and $0.4 \mathrm{MeV}$. Therefore we conclude that, in terms of convergence, the UCOM potential from Ref. 35] and the SRG $\lambda=3.0 \mathrm{fm}^{-1}$ potential are roughly equivalent.

\section{Running of Ground-State Energies}

The SRG flow equation guarantees a unitary transformed Hamiltonian and exactly the same values for observables only if the entire Hamiltonian is kept. However, the commutators in Eq. (2) generate many-body interactions as $\lambda$ is lowered (e.g., substitute second-quantized operators) and therefore in practice there will always be a truncation. But this is also true of the initial EFT Hamiltonian, which will already have many-body interactions to all orders that evolve with $\lambda$. However, there is a chiral EFT power-counting hierarchy 


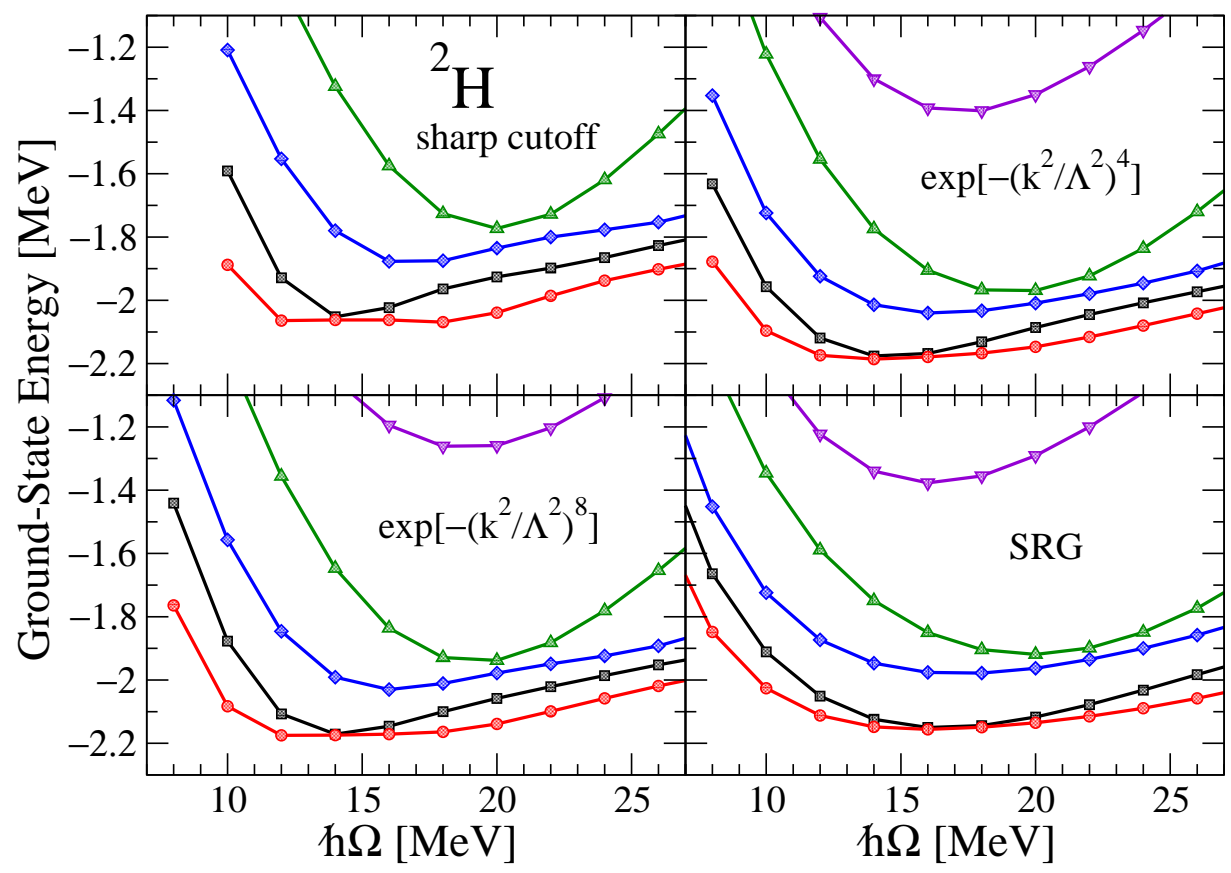

Fig. 9. Ground-state energy of the deuteron as a function of $\hbar \Omega$ for a sharp and two smooth $V_{\text {low } k}$ potentials with $\Lambda=2 \mathrm{fm}^{-1}$ and an SRG potential with $\lambda=2 \mathrm{fm}^{-1}$. The initial potential is the $500 \mathrm{MeV} \mathrm{N}{ }^{3} \mathrm{LO}$ NN-only potential from Ref. [13]. The legend from Fig. 1 applies here.

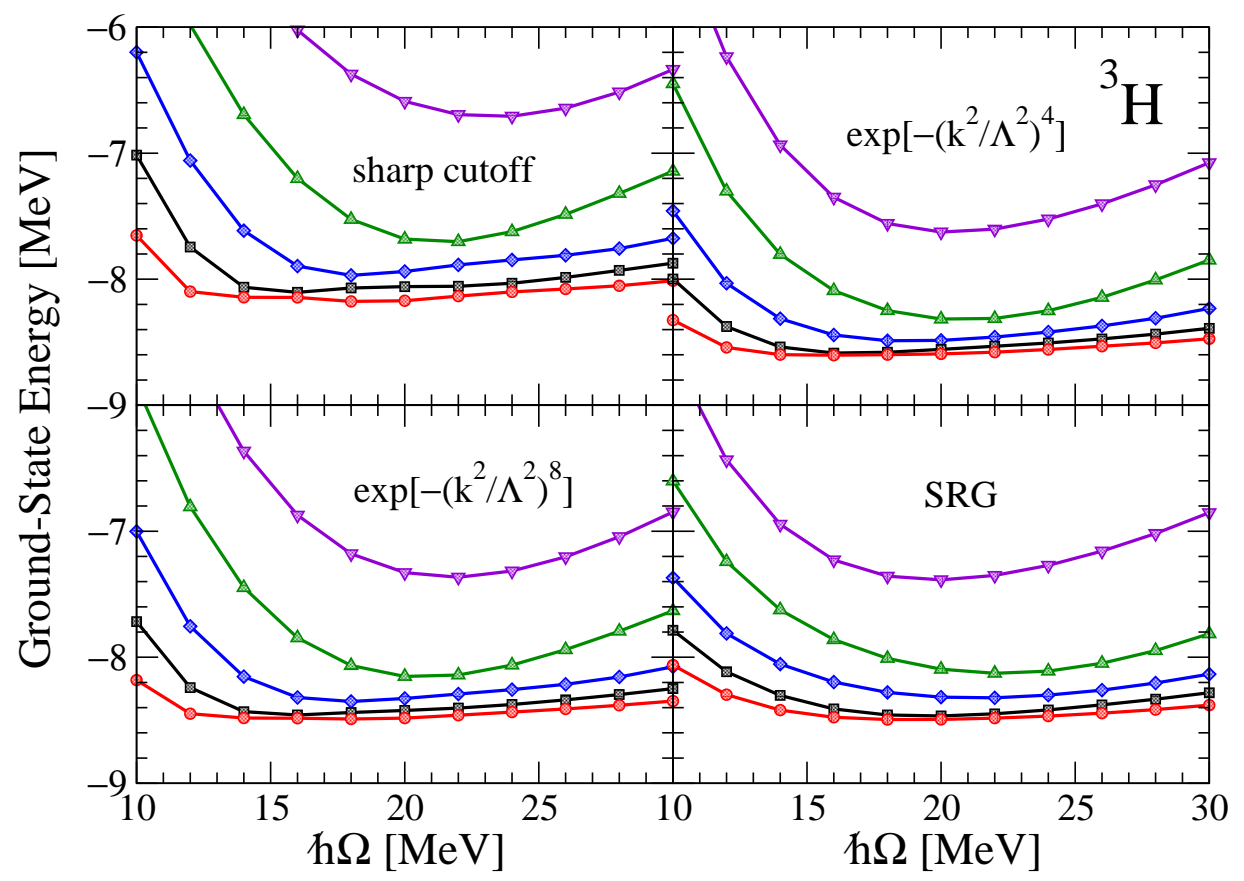

Fig. 10. Ground-state energy of the triton as a function of $\hbar \Omega$ for a sharp and two smooth $V_{\text {low } k}$ potentials with $\Lambda=2 \mathrm{fm}^{-1}$ and an SRG potential with $\lambda=2 \mathrm{fm}^{-1}$. The initial potential is the $500 \mathrm{MeV} \mathrm{N}^{3} \mathrm{LO}$ NN-only potential from Ref. [13. The legend from Fig. 1 applies here. 


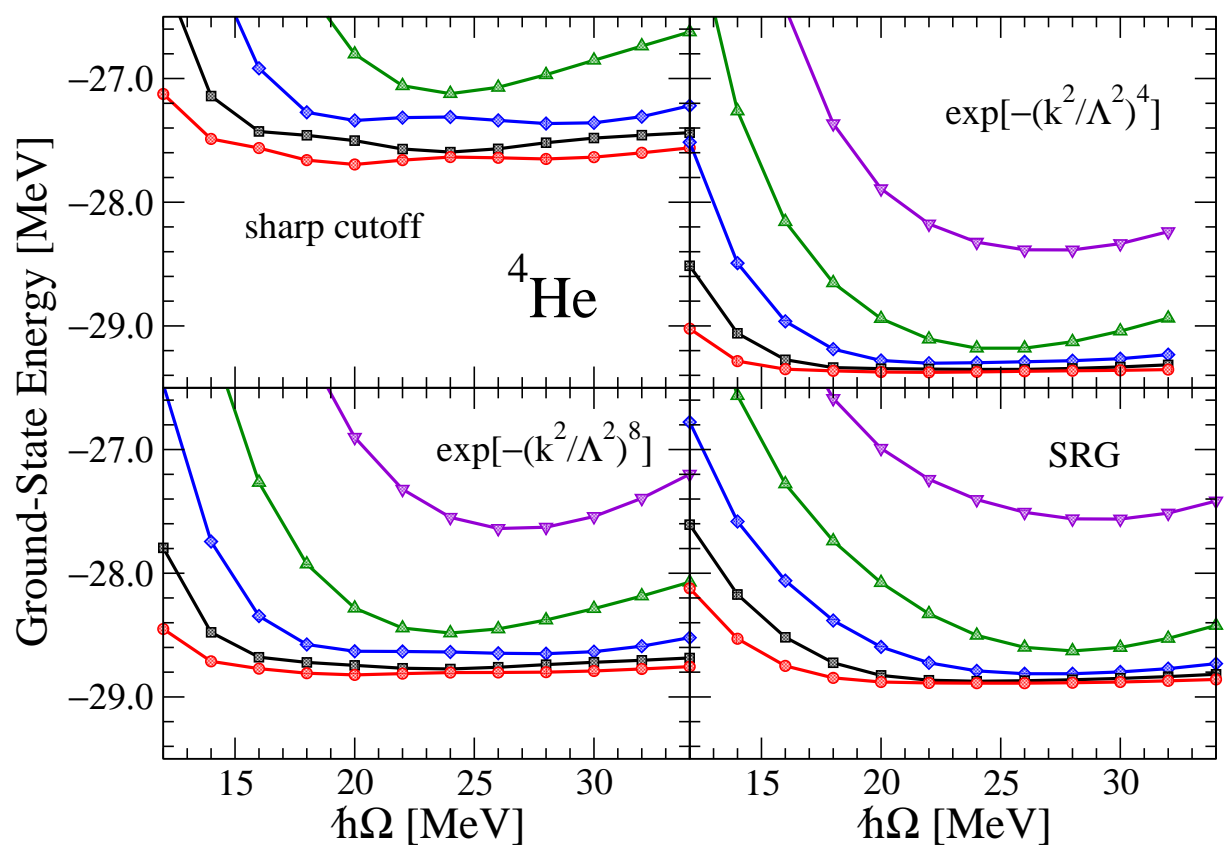

Fig. 11. Ground-state energy of ${ }^{4} \mathrm{He}$ as a function of $\hbar \Omega$ for a sharp and two smooth $V_{\text {low } k}$ potentials with $\Lambda=2 \mathrm{fm}^{-1}$ and an SRG potential with $\lambda=2 \mathrm{fm}^{-1}$. The initial potential is the $500 \mathrm{MeV} \mathrm{N}{ }^{3} \mathrm{LO}$ NN-only potential from Ref. [13. The legend from Fig. 1 applies here.

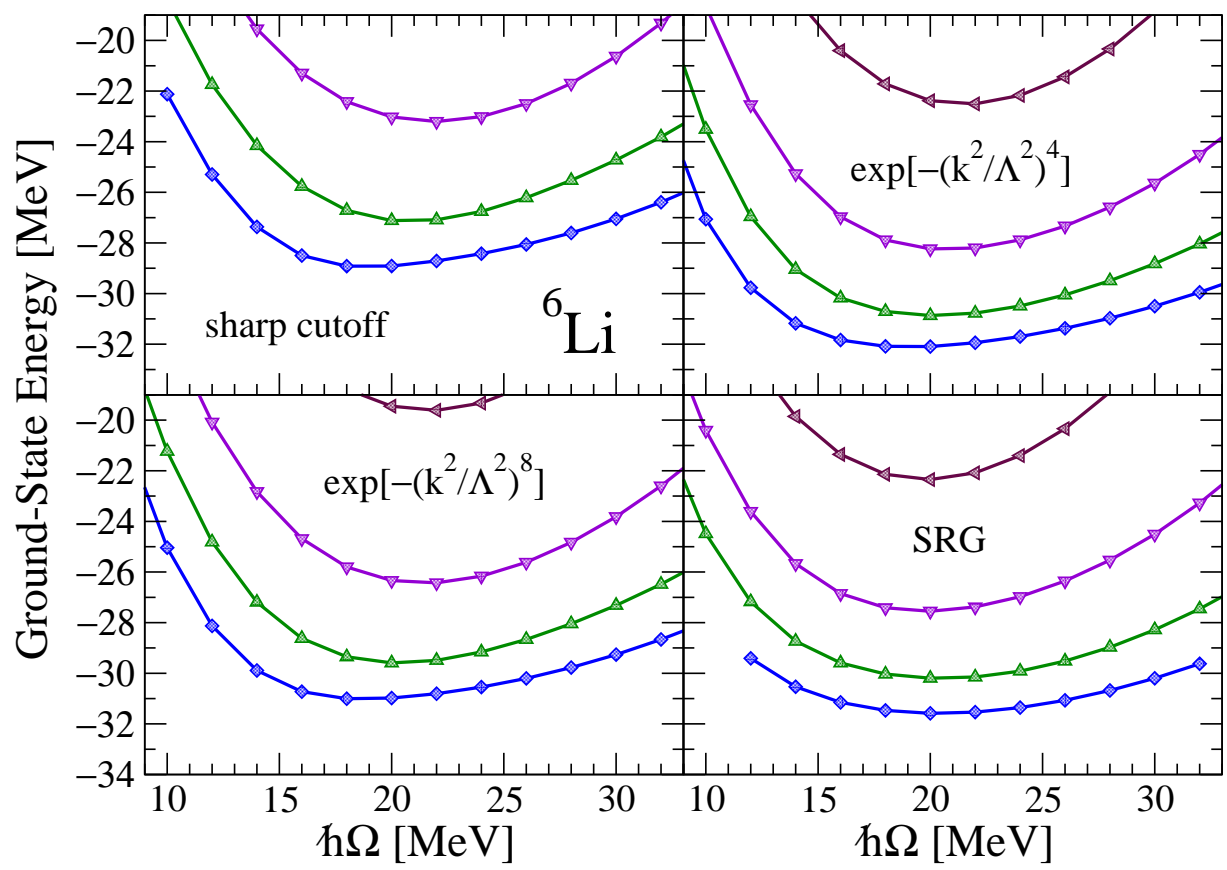

Fig. 12. Ground-state energy of ${ }^{6} \mathrm{Li}$ as a function of $\hbar \Omega$ for a sharp and two smooth $V_{\text {low } k}$ potentials with $\Lambda=2 \mathrm{fm}^{-1}$ and an SRG potential with $\lambda=2 \mathrm{fm}^{-1}$. The initial potential is the $500 \mathrm{MeV} \mathrm{N}{ }^{3} \mathrm{LO}$ NN-only potential from Ref. [13. The legend from Fig. 1 applies here. 
that establishes natural sizes for the contributions from many-body forces. This enables truncation at few-body interactions with a controlled error (at $\mathrm{N}^{3} \mathrm{LO}$ this includes a long-range four-body interaction). Therefore the key to the usefulness of the SRG for nuclear structure is maintaining this hierarchy with a comparable truncation error. In the present calculations, keeping only two-body interactions means that the transformations are only approximately unitary for $A \geqslant 3$ and few-body binding energies will change ("run") with $\lambda$. By tracking this running, we can determine the expected net contributions from many-body forces in a more complete calculation. The useful range of $\lambda$ is where the variations are of natural size (i.e., the same order as the EFT truncation errors).

For the lower $\lambda$ 's in the lighter nuclei, our predictions for ground-state energies are fully converged. However, in other cases we need to extrapolate the energies to $N_{\max }=\infty$. If $\alpha$ labels the $\hbar \Omega$ values and $i$ the $N_{\max }$ values for each $\alpha$, a possible model for ground-state energies is

$$
E_{\alpha i}=E_{\infty}+A_{\alpha} e^{-b_{\alpha} N_{i}}
$$

where $A_{\alpha}$ and $b_{\alpha}$ are constants. The goal of a fit to calculations such as those in Section 2 is to determine the common parameter $E_{\infty}$, which is the estimate for the ground-state energy extrapolated to $N_{\max }=\infty$. Presumably this approach will work better with larger nuclei for which $N_{\max }$ may become a good logarithmic measure of the number of states.

Given the model in Eq. (4), one way to proceed is to minimize the function

$$
f\left(E_{\infty},\left\{A_{\alpha}\right\},\left\{b_{\alpha}\right\}\right)=\sum_{\alpha, i}\left(E_{\alpha i}-E_{\infty}-A_{\alpha} e^{-b_{\alpha} N_{i}}\right)^{2} / \sigma_{\alpha i}^{2}
$$

where we have allowed the possibility for different weights depending on $\hbar \Omega$ and $N_{\max }$. As posed this is a nonlinear least-squares minimization problem with many parameters, for which robust solutions are difficult to find. An alternative approach is to recast the problem so that all parameters except $E_{\infty}$ are treated as linear parameters. This is possible because our problem is strictly variational, so $E_{\alpha i}-E_{\infty}>0$ for all $\alpha$ and $i$, which means that the logarithm of Eq. (4) is well defined. So one considers instead

$$
\log \left(E_{\alpha i}-E_{\infty}\right)=\log A_{\alpha}-b_{\alpha} N_{i} \equiv a_{\alpha}-b_{\alpha} N_{i}
$$

For each value of $E_{\infty}$, this is a linear least-squares problem in the $\left\{a_{\alpha}\right\}$ and $\left\{b_{\alpha}\right\}$ parameters. Thus we have a one-dimensional constrained minimization problem with the function

$$
g\left(E_{\infty}\right)=\sum_{\alpha, i}\left(\log \left(E_{\alpha i}-E_{\infty}\right)-a_{\alpha}-b_{\alpha} N_{i}\right)^{2} / \sigma_{\alpha i}^{2}
$$




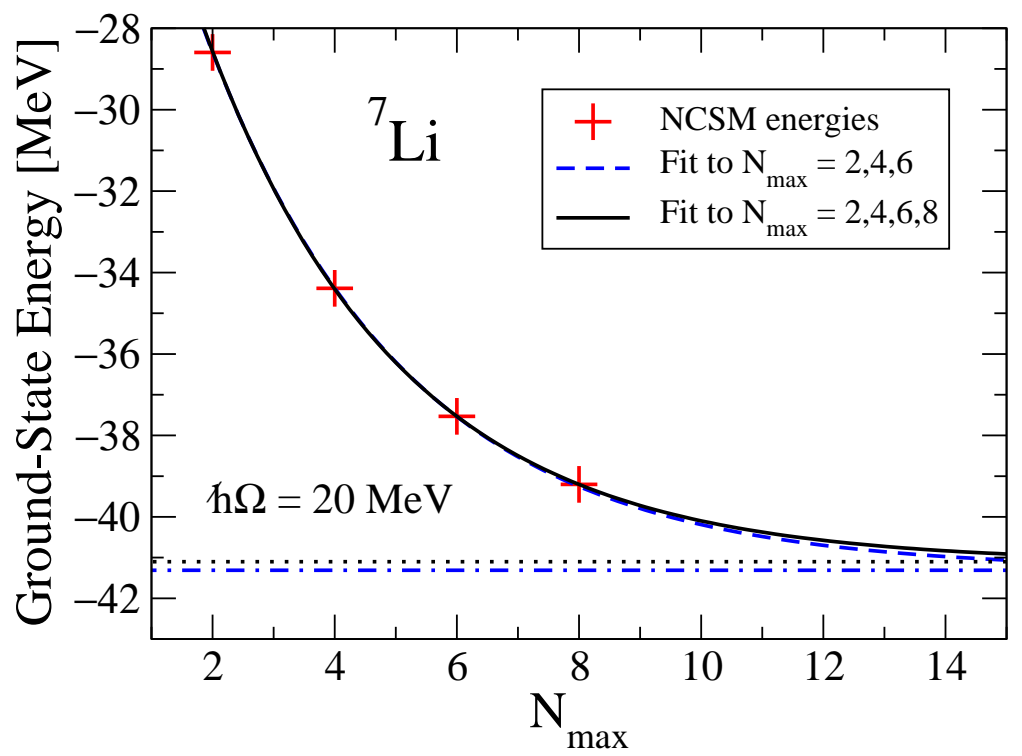

Fig. 13. Fits to the NCSM ground-state energies for ${ }^{7} \mathrm{Li}$ at $\lambda=2 \mathrm{fm}^{-1}$ with $\hbar \Omega$ $=20 \mathrm{MeV}$ according to the model in Eq. (4), using the procedure described in the text. The dashed line uses the NCSM energies (pluses) up to $N_{\max }=6$, with the extrapolated value of $E_{\infty}$ shown as the dot-dash line, while the solid line uses up to $N_{\max }=8$, with the dotted line the extrapolated value of $E_{\infty}$.

where the $\left\{a_{\alpha}\right\}$ and $\left\{b_{\alpha}\right\}$ are determined directly within the function $g$ by invoking a constrained linear least-squares minimization routine. The constraint is the bound $E_{\infty} \leqslant \min \left(\left\{E_{\alpha i}\right\}\right)$, where $E_{\infty}<0$ and "min" means "most negative". Again, we allow for weights depending on $N_{\max }$ and/or $\hbar \Omega$.

In the present investigation, we apply Eq. (7) with only the $\hbar \Omega$ value that yields the lowest energy in the largest space, weighting different $N_{\text {max }}$ by the slope of the energy vs. $N_{\max }$, and using the spread of results from neighboring $\hbar \Omega$ values to determine a conservative confidence interval for the extrapolation. This procedure is applied for all results in this section. An example of the fit to NCSM data is given in Fig. 13. A more complete and systematic study of extrapolation in the NCSM will be given in Ref. [33].

The extrapolated results for ground-state energies (in $\mathrm{MeV}$ ) are summarized in Table 1 along with the value of $\hbar \Omega$ (in $\mathrm{MeV}$ ) for which the energy is minimal in the largest spaces. The uncertainties represent conservative confidence intervals. When not given, the result should be accurate to the digits displayed. The ${ }^{3} \mathrm{H}$ and ${ }^{4} \mathrm{He}$ results are fully converged for $\lambda \leqslant 2 \mathrm{fm}^{-1}$. For larger $\lambda$ values the ${ }^{3} \mathrm{H}$ energies are evaluated in a large basis $\left(N_{\max }=48\right)$ to ensure convergence for all $\lambda$ and the ${ }^{4} \mathrm{He}$ results are extrapolated. All of the results for $A>4$ are extrapolated to some degree, with increasingly small extrapolations with decreasing $\lambda$ (and energies for the bare potentials could not be reliably extrapolated in the spaces used). 
Table 1

Ground-state energies and optimal $\hbar \Omega$ (in $\mathrm{MeV}$ ) of light nuclei for SRG-evolved potentials. When an uncertainty is given it represents a confidence interval derived from an extrapolation to $N_{\max }=\infty$. Otherwise the energy is converged to the digits shown.

\begin{tabular}{|l|cc|cc|cc|cc|cc|}
\hline \multicolumn{1}{|c|}{} & \multicolumn{2}{|c|}{${ }^{3} \mathrm{H}$} & \multicolumn{2}{|c|}{${ }^{4} \mathrm{He}$} & \multicolumn{2}{|c|}{${ }^{6} \mathrm{He}$} & \multicolumn{2}{|c|}{${ }^{6} \mathrm{Li}$} & \multicolumn{2}{c|}{${ }^{7} \mathrm{Li}$} \\
\hline$\infty$ & $\hbar \Omega$ & $E_{\mathrm{gs}}$ & $\hbar \Omega$ & $E_{\mathrm{gs}}$ & $\hbar \Omega$ & $E_{\mathrm{gs}}$ & $\hbar \Omega$ & $E_{\mathrm{gs}}$ & $\hbar \Omega$ & $E_{\mathrm{gs}}$ \\
3.0 & - & -7.85 & 42 & $-26.1(8)$ & & & & & & \\
2.5 & 24 & -8.29 & 34 & $-27.5(3)$ & 28 & $-28(1)$ & 28 & $-31.5(8)$ & 24 & $-38.7(30)$ \\
2.25 & 22 & -8.47 & 28 & $-28.2(2)$ & 24 & $-28.9(3)$ & 24 & $-32.1(3)$ & 24 & $-38.7(20)$ \\
2.0 & 18 & -8.53 & 24 & $-28.6(1)$ & 22 & $-29.4(2)$ & 22 & $-32.5(2)$ & 22 & $-40.3(10)$ \\
1.75 & 16 & -8.55 & 20 & -29.13 & 16 & -30.6 & 18 & -33.6 & 18 & $-41.7(4)$ \\
1.5 & 12 & -8.48 & 18 & -28.86 & 14 & -30.7 & 16 & -33.7 & 16 & $-42.0(3)$ \\
1.25 & 10 & -8.21 & 14 & -27.58 & 12 & -29.9 & 12 & -32.9 & 12 & $-41.1(2)$ \\
1.0 & 8 & -7.63 & 14 & -24.80 & 10 & -27.4 & 10 & -30.4 & 12 & $-37.8(2)$ \\
\hline
\end{tabular}

If we calculate the energy of a nucleus using the SRG-evolved NN interaction only, the running of the energy is the running of the net three-body (and higher-body) contribution to the energy. Over a wide range of cutoffs, manybody interactions generated in the $R G$ will be the same scale as the starting $3 \mathrm{~N}$ interactions in the chiral EFT. Figures 14 to 16 show the NN-only running for ${ }^{3} \mathrm{H},{ }^{4} \mathrm{He},{ }^{6} \mathrm{He}$, and ${ }^{6} \mathrm{Li}$. The energy plotted is either the converged value or extrapolated from the best $\hbar \Omega$ with an error bar (i.e., a confidence interval) as described above. Figures 17 to 20 are binding-energy correlation plots between various nuclei.

The pattern of SRG running for ${ }^{4} \mathrm{He},{ }^{6} \mathrm{He},{ }^{6} \mathrm{Li}$, and ${ }^{7} \mathrm{Li}$ is qualitatively similar to the pattern for ${ }^{3} \mathrm{H}$ shown in Fig. 14. That is, a slow increase in the binding energy as $\lambda$ decreases to $\lambda=1.5-1.8 \mathrm{fm}^{-1}$ and then a steep decrease. The two SRG curves in Fig. 14 show the difference between evolving the neutron-proton interaction only and evolving the full NN interaction. The SRG pattern is also qualitatively similar to the running for $V_{\text {low } k}$ potentials [8,5]. As observed for $V_{\text {low } k}$ [8,4], the size of three-body contributions is natural according to chiral EFT power counting. This is true for the net energy within the entire range of $\lambda$ shown in the figures, but future calculations are needed to investigate whether the individual contributions from long-range and short-range $3 \mathrm{~N}$ forces remain natural. The smoothness of the running adds credibility to the extrapolations and the associated estimates of confidence intervals; when the estimate has been improved (e.g., from a larger $N_{\max }$ result), the running and correlation plots have become more regular. 


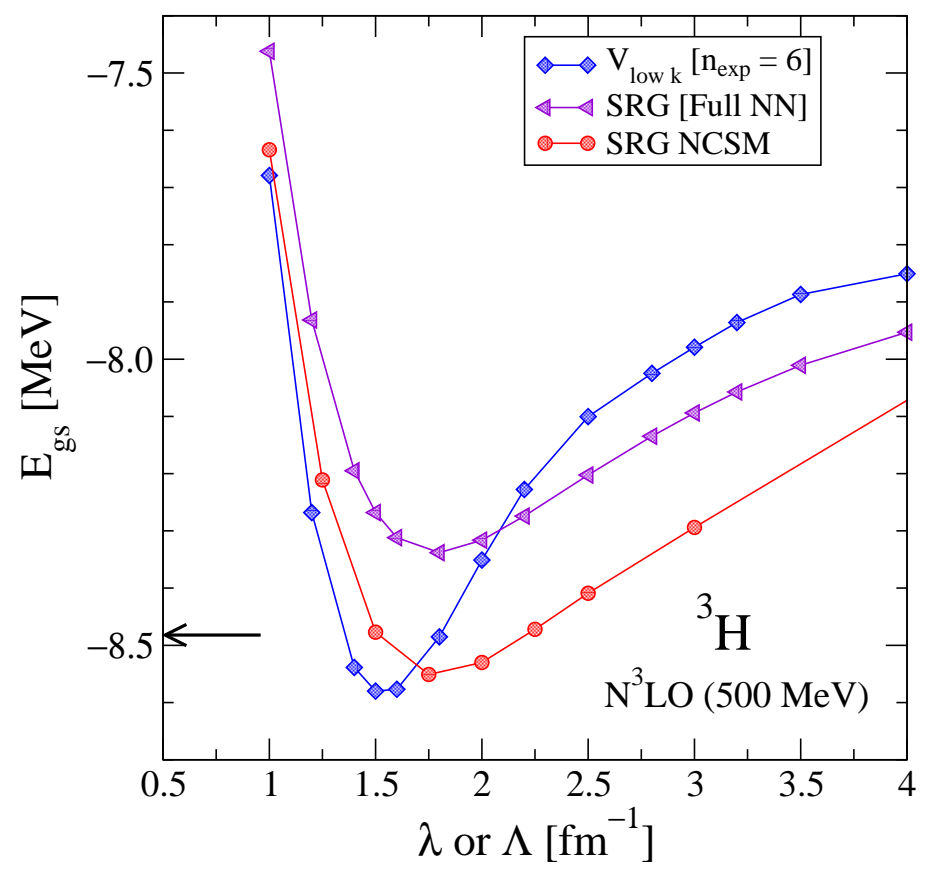

Fig. 14. Plot of the ground-state energy of the triton vs. $\lambda$ (if SRG) or $\Lambda$ (if $V_{\text {low } k}$ ) for potentials evolved from the $500 \mathrm{MeV} \mathrm{N}{ }^{3} \mathrm{LO}$ NN-only potential from Ref. [13. The arrow marks the experimental binding. The two SRG curves show the difference between evolving the full $\mathrm{NN}$ interaction and evolving the neutron-proton interaction only.
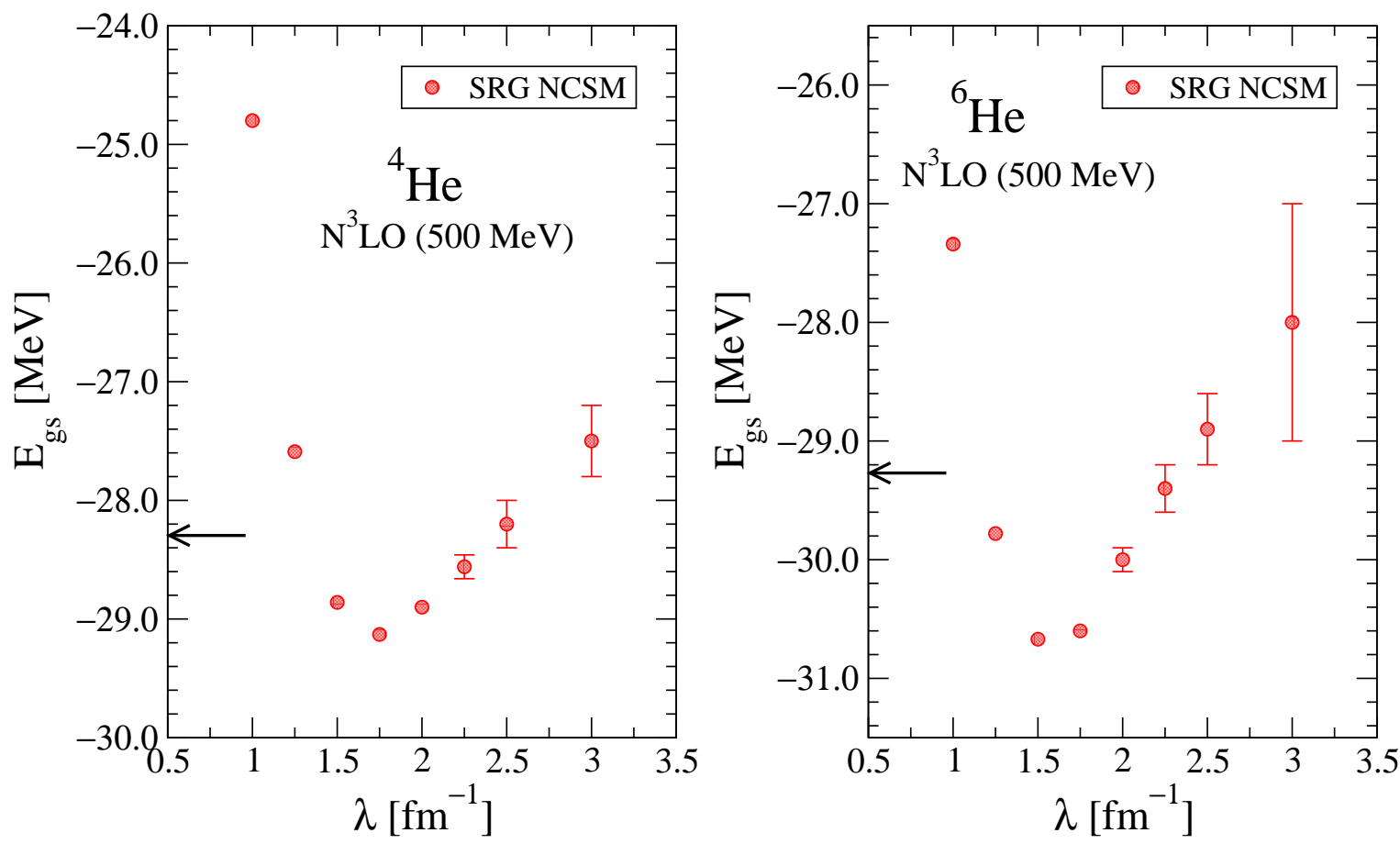

Fig. 15. Plot of the ground-state energy of ${ }^{4} \mathrm{He}$ and ${ }^{6} \mathrm{He}$ vs. $\lambda$ for potentials evolved by the SRG from the $500 \mathrm{MeV} \mathrm{N}{ }^{3} \mathrm{LO}$ NN-only potential from Ref. [13]. Conservative error bars have been included with the larger $\lambda$ 's, for which an extrapolation is needed. The arrow marks the experimental binding. 

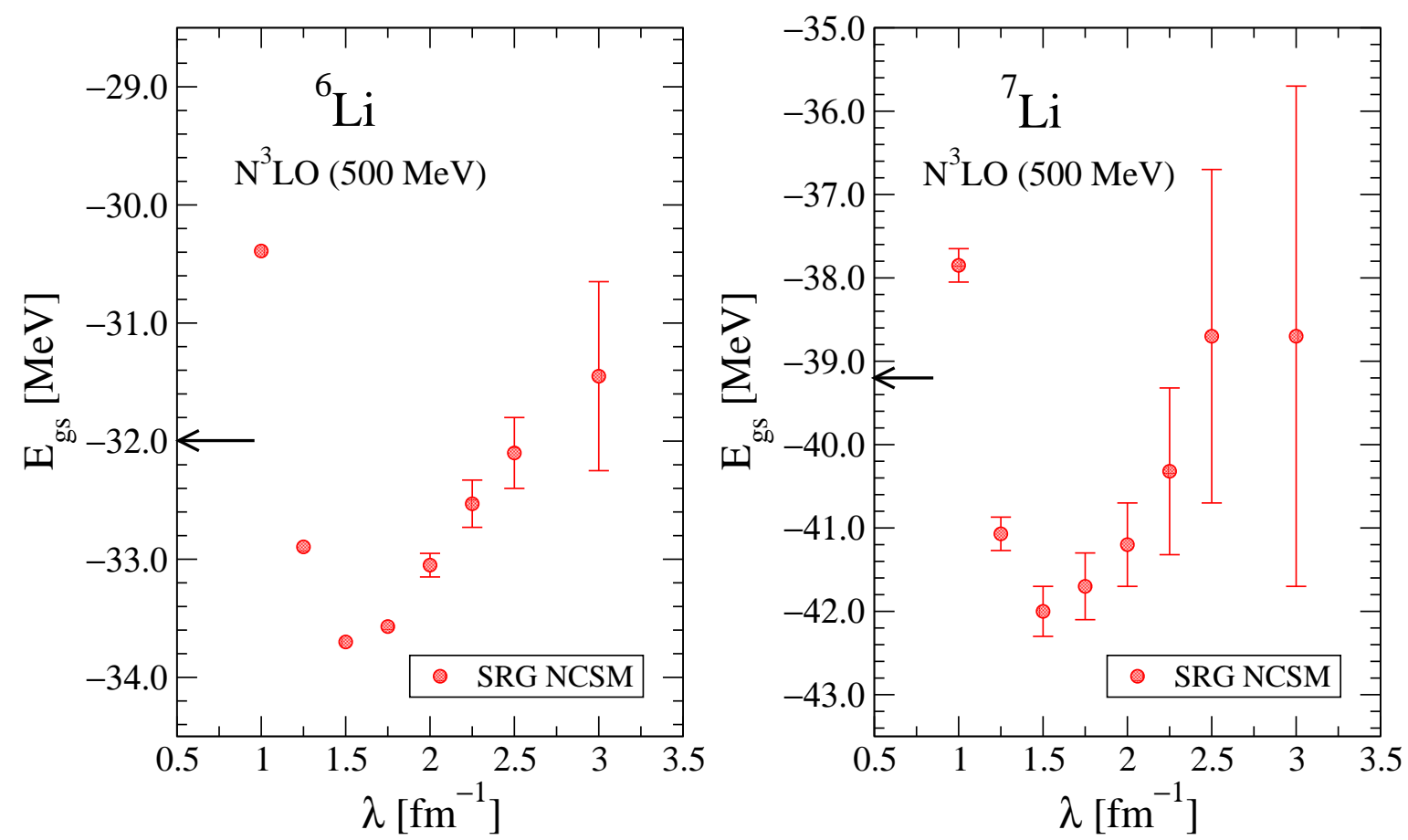

Fig. 16. Plot of the ground-state energy of ${ }^{6} \mathrm{Li}$ and ${ }^{7} \mathrm{Li}$ vs. $\lambda$ for $\mathrm{SRG}$ potentials evolved from the $500 \mathrm{MeV} \mathrm{N}{ }^{3} \mathrm{LO}$ NN-only potential from Ref. [13. Conservative error bars have been included with the larger $\lambda$ 's, for which an extrapolation is needed. The arrow marks the experimental binding.

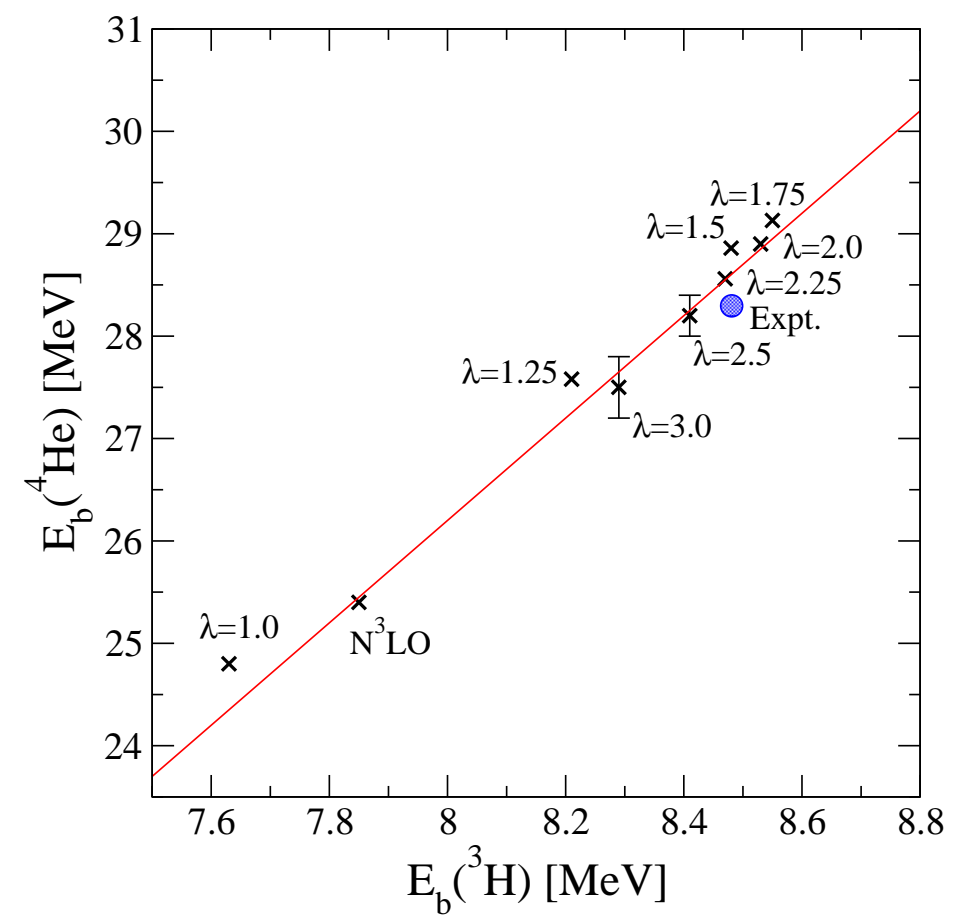

Fig. 17. Tjon line for SRG potentials evolved from the $500 \mathrm{MeV} \mathrm{N}{ }^{3} \mathrm{LO}$ NN-only potential from Ref. [13] (with $\lambda$ in $\mathrm{fm}^{-1}$ ). Conservative error bars have been included with the larger $\lambda$ 's, for which an extrapolation is needed. The result for ${ }^{4} \mathrm{He}$ with the bare potential is from Ref. [36]. The line is a fit to NN potentials from Ref. [37]. 


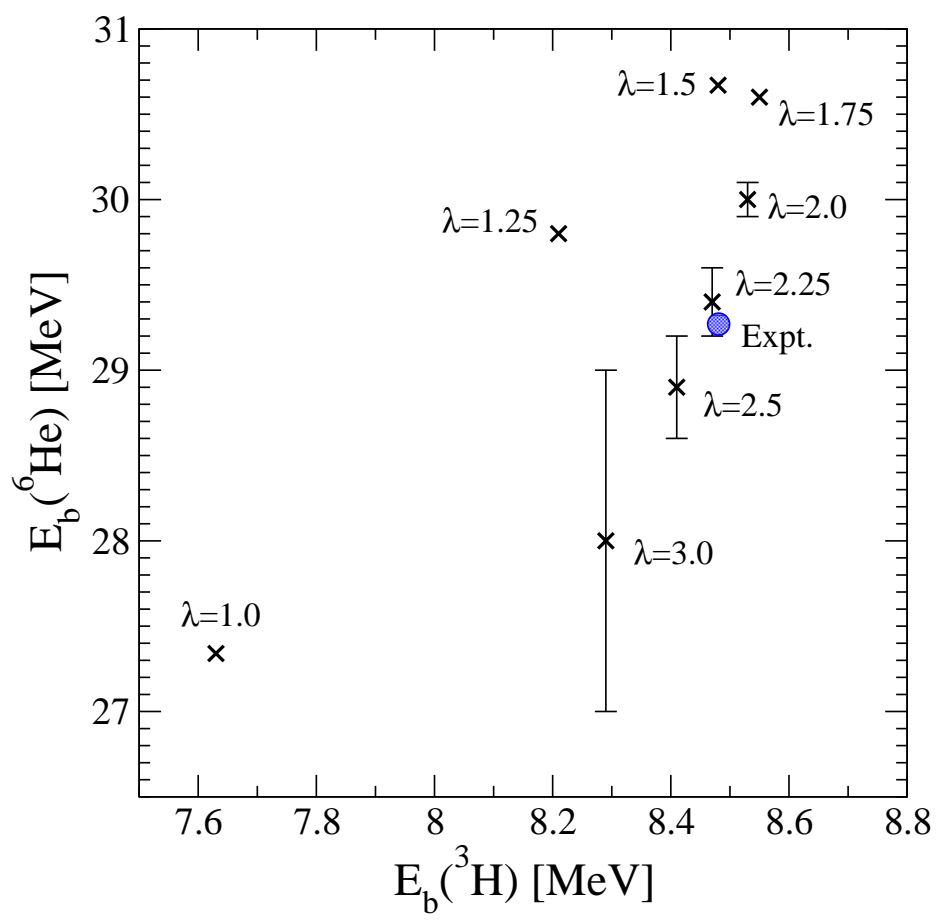

Fig. 18. Binding energy correlation plot between ${ }^{6} \mathrm{He}$ and ${ }^{3} \mathrm{H}$ for SRG potentials evolved from the $500 \mathrm{MeV} \mathrm{N}^{3} \mathrm{LO}$ NN-only potential from Ref. [13] (with $\lambda$ in $\mathrm{fm}^{-1}$ ). Conservative error bars have been included with the larger $\lambda$ 's, for which an extrapolation is needed.

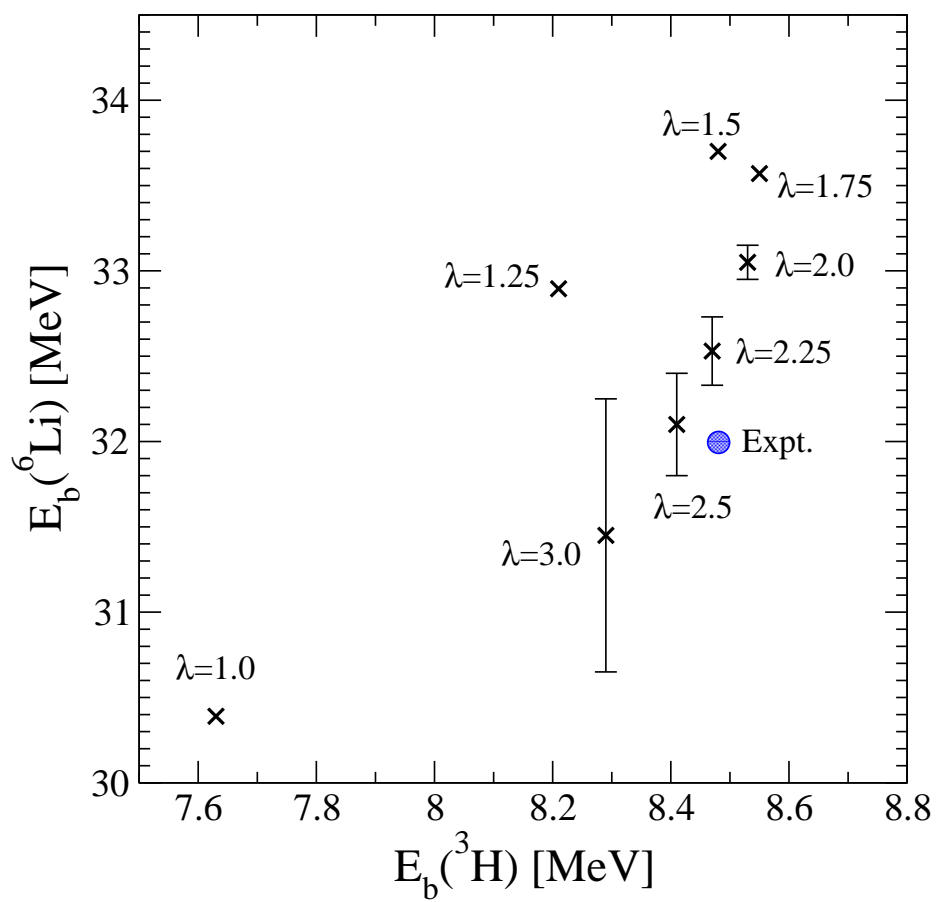

Fig. 19. Binding energy correlation plot between ${ }^{6} \mathrm{Li}$ and ${ }^{3} \mathrm{H}$ for SRG potentials evolved from the $500 \mathrm{MeV} \mathrm{N}^{3} \mathrm{LO}$ NN-only potential from Ref. [13] (with $\lambda$ in $\mathrm{fm}^{-1}$ ). Conservative error bars have been included with the larger $\lambda$ 's, for which an extrapolation is needed. 


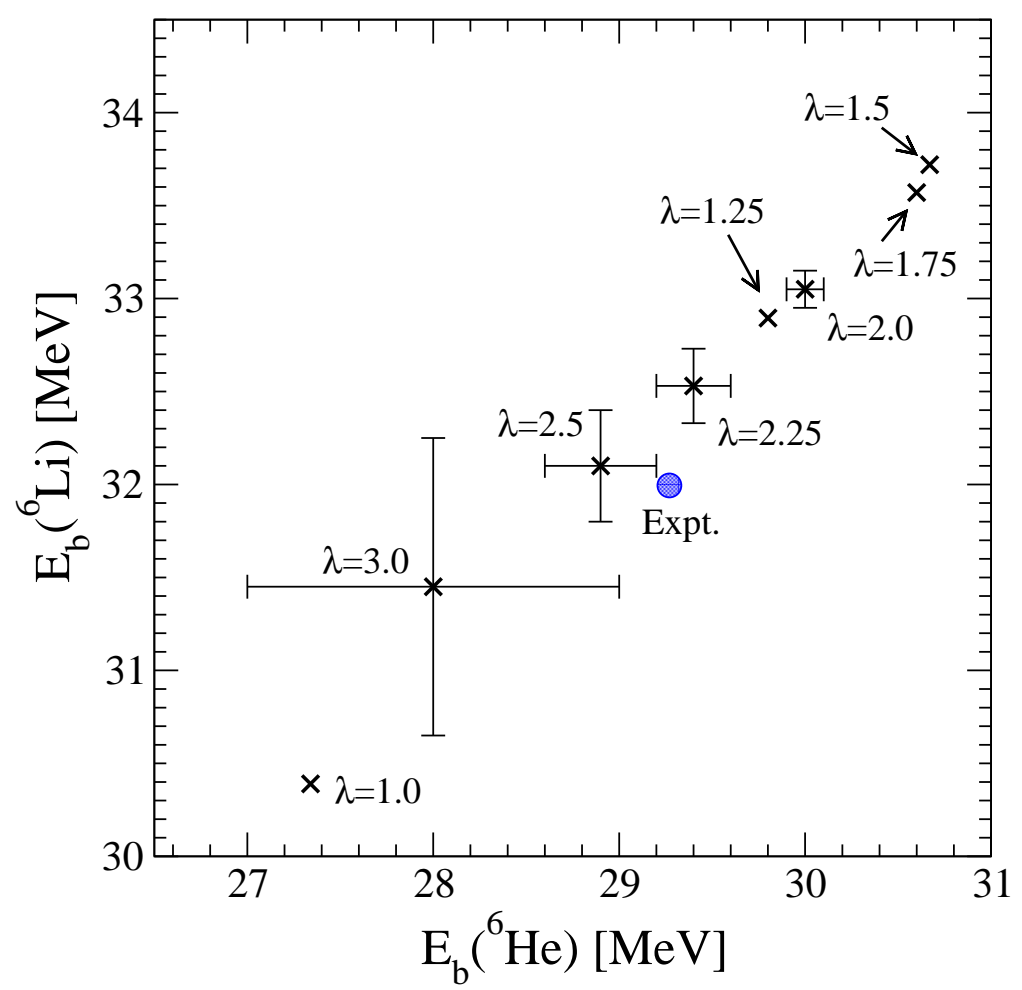

Fig. 20. Binding energy correlation plot between ${ }^{6} \mathrm{Li}$ and ${ }^{6} \mathrm{He}$ for SRG potentials evolved from the $500 \mathrm{MeV} \mathrm{N}^{3} \mathrm{LO} \mathrm{NN}$-only potential from Ref. [13] (with $\lambda$ in fm${ }^{-1}$ ). Conservative error bars have been included with the larger $\lambda$ 's, for which an extrapolation is needed.

The correlation plot for ${ }^{4} \mathrm{He}$ vs. ${ }^{3} \mathrm{H}$ shows the expected movement along the Tjon line, with a slight loop closing to the left as $\lambda$ decreases. The latter behavior is amplified in the ${ }^{6} \mathrm{He}$ vs. ${ }^{3} \mathrm{H}$ and ${ }^{6} \mathrm{Li}$ vs. ${ }^{3} \mathrm{H}$ plots. The plot of ${ }^{6} \mathrm{Li}$ vs. ${ }^{6} \mathrm{He}$ is very close to linear. Tjon lines are also found in coupled cluster calculations of ${ }^{15} \mathrm{O},{ }^{16} \mathrm{O},{ }^{17} \mathrm{O},{ }^{15} \mathrm{~N}$, and ${ }^{17} \mathrm{~F}$ [38]. From these plots one sees some $\lambda$ 's for which predicted ground-state energies are quite close to experiment (e.g., $\lambda=2.25 \mathrm{fm}^{-1}$ ). These may be good choices for calculations for which small net three-body contributions are desirable. However, we emphasize that this is not a global trend. Indeed, nuclear matter does not saturate with only NN interactions at such $\lambda$ 's (except perhaps at very large density). Thus calculations in heavier nuclei (e.g., ${ }^{16} \mathrm{O},{ }^{40} \mathrm{Ca}, \ldots$ ) will be increasingly overbound.

\section{Other Observables}

In this section, we show some limited results of excitation energies and radii for ${ }^{7} \mathrm{Li}$. Because we expect sensitivity to three-body contributions, we include these results primarily to assess convergence trends as a baseline for future $3 \mathrm{~N}$ calculations and to compare to previous results from Ref. [18]. 


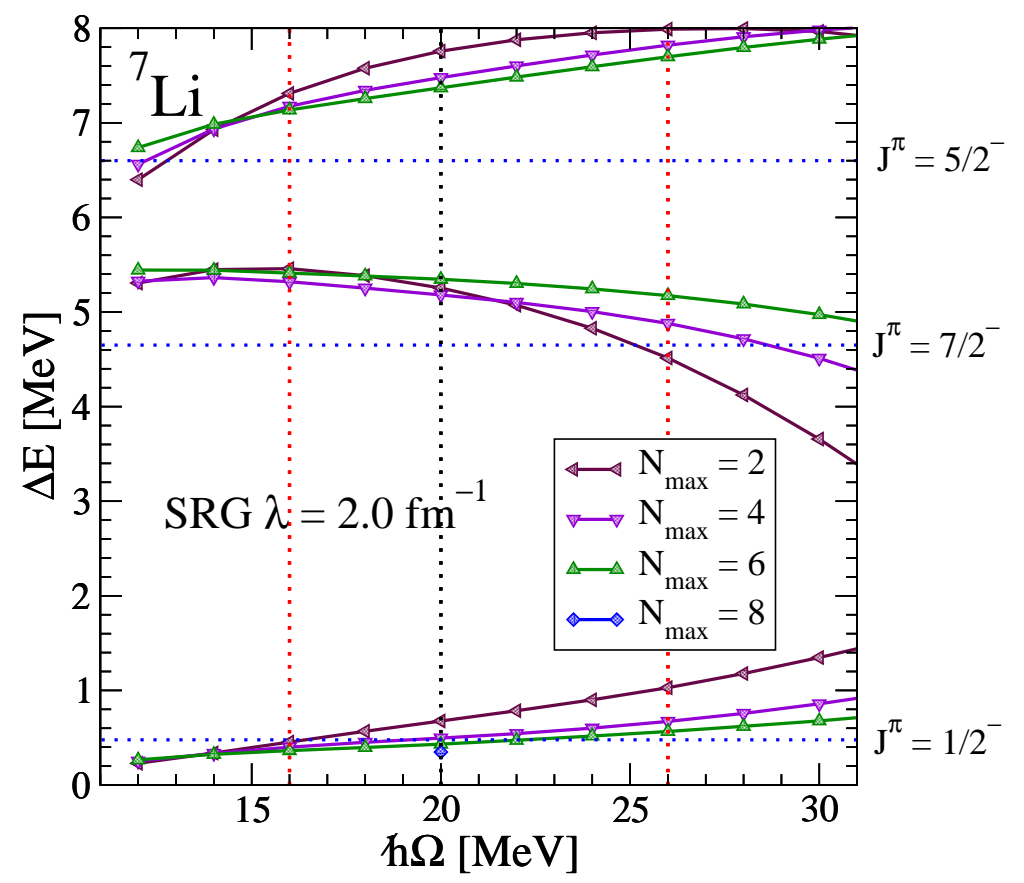

Fig. 21. Excitation energies of the lowest natural-partiy states of ${ }^{7} \mathrm{Li}$ as a function of $\hbar \Omega$ for $\lambda=2.0 \mathrm{fm}^{-1}$. The initial potential is the $500 \mathrm{MeV} \mathrm{N}{ }^{3} \mathrm{LO}$ NN-only potential from Ref. [13. The horizontal dotted lines are the experimental values while the vertical dotted lines mark the optimal $\hbar \Omega$ value for the ground-state energy (middle) and the range for which estimates are close to this.

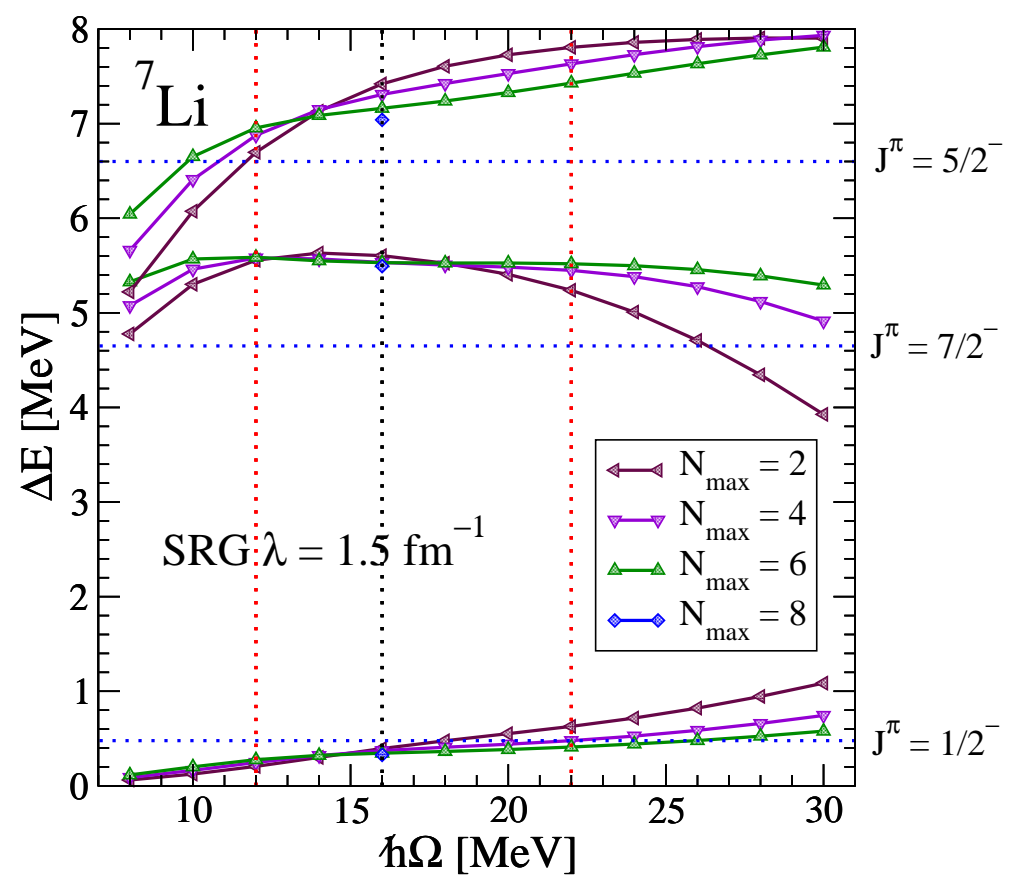

Fig. 22. Excitation energies of the lowest natural-parity states of ${ }^{7} \mathrm{Li}$ as a function of $\hbar \Omega$ for $\lambda=1.5 \mathrm{fm}^{-1}$. The initial potential is the $500 \mathrm{MeV} \mathrm{N}{ }^{3} \mathrm{LO}$ NN-only potential from Ref. [13]. The horizontal dotted lines are the experimental values while the vertical dotted lines mark the optimal $\hbar \Omega$ value for the ground-state energy (middle) and the range for which estimates are close to this. 
The spectrum of low-lying natural-parity excited states in ${ }^{7} \mathrm{Li}$ is shown in Figs. 21 and 22 for $\lambda=2.0 \mathrm{fm}^{-1}$ and $1.5 \mathrm{fm}^{-1}$. The excitation energy is plotted against $\hbar \Omega$ for different $N_{\max }$ values to facilitate comparison to similar plots from NCSM Lee-Suzuki calculations based on the same potential [18]. The vertical dotted lines mark the range of $\hbar \Omega$ that has the lowest ground-state energy estimates, with the middle line marking the best energy estimate for the ground state. The higher $N_{\text {max }}$ predictions are flatter as a function of $\hbar \Omega$, as expected, and $\lambda=1.5 \mathrm{fm}^{-1}$ converges faster than $\lambda=2.0 \mathrm{fm}^{-1}$. However, we observe that the curves are not as flat as in the corresponding plot in Ref. [18]. The spread from $N_{\max }=2$ to $N_{\max }=6$ is not large, but also appears larger than in Ref. [18].

In Figs. 23 and 24, we plot the point proton radii of ${ }^{4} \mathrm{He}$ and ${ }^{6} \mathrm{Li}$ as a function of $\hbar \Omega$, with the different $N_{\max }$ curves having the same legends as in Figs. 1 and 5. These plots are made using the bare operator for $r^{2}$ at all $\lambda$, rather than an evolved operator. (Note that $r^{2}$ for the proton is not an observable.) Since $r^{2}$ samples primarily long distances, its matrix elements depend weakly on the cutoff [5]. For the deuteron, the rms radius changes from the $\lambda=\infty$ value by less than $1 \%$ by $\lambda=1.5 \mathrm{fm}^{-1}$ and by about $4 \%$ by $\lambda=1.0 \mathrm{fm}^{-1}$. Our observations are consistent with the investigations reported recently on the range-dependence of another renormalization scheme [39].

For ${ }^{4} \mathrm{He}$, we observe that with decreasing $\lambda$ the curves for a given $N_{\text {max }}$ get flatter; for $\lambda=1.5 \mathrm{fm}^{-1}$ our $N_{\max }=12$ results are essentially independent of $\hbar \Omega$. The different $N_{\max }$ curves cross each other in a decreasing range of $\hbar \Omega$ as $\lambda$ decreases. For $\lambda=1.5 \mathrm{fm}^{-1}$, all of the curves for both nuclei intersect at the same point. If we consider the intersection of the three highest $N_{\max }$ results, it is close to (but generally slightly below) the $\hbar \Omega$ for the lowest groundstate energy estimate. A similar observation has been reported for the NCSM Lee-Suzuki calculations in Ref. [18]. The trend is consistent with the idea that if you have the optimal $\hbar \Omega$ for a given nucleus (which scales with $A^{-1 / 3}$ and inversely with the average radius squared), then the radius should be minimally sensitive to changes in the basis size. For ${ }^{6} \mathrm{Li}$, the intersection point is well below the optimal $\hbar \Omega$ for the energy except at very low cutoffs; the radii for larger $\lambda$ are not converged in the largest spaces considered here.

In Fig. 24, the dotted lines indicate the radii from the NCSM Lee-Suzuki calculations of the bare $\mathrm{N}^{3} \mathrm{LO}$ potential without $3 \mathrm{~N}$ interactions (middle) and with two different 3N fits (top and bottom) [18. The spread of these predictions can be taken as an estimate of the expected $3 \mathrm{~N}$ contribution for various $\lambda$ 's. The $\hbar \Omega$ dependence here is steep, but if we take the intersection point to determine the NN radius, then the results for $\lambda=3,2,1.5 \mathrm{fm}^{-1}$ are all compatible with the radii from Ref. [18. Moreover, the trend in the radius with decreasing $\lambda$ parallels the running of the energy with $\lambda$ (see Fig. 16). 

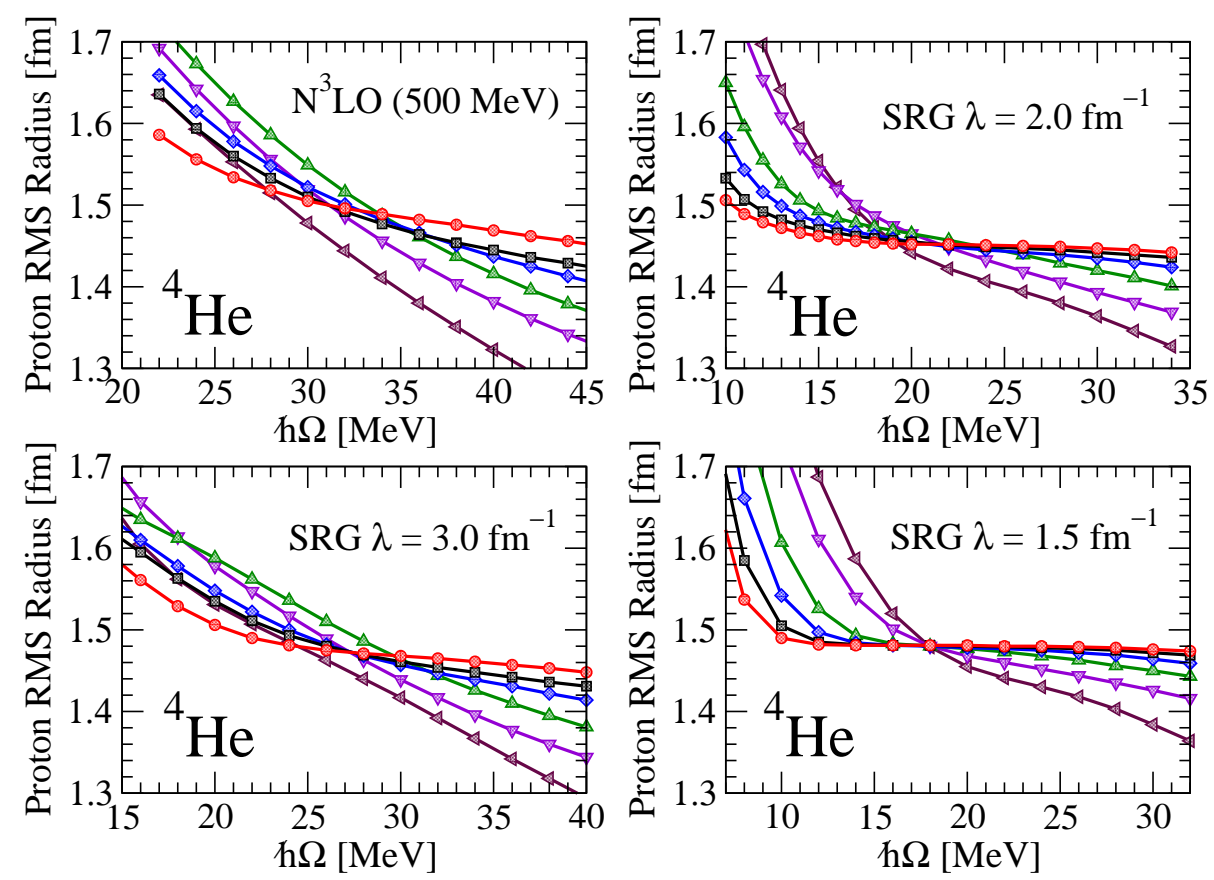

Fig. 23. Point proton radii of ${ }^{4} \mathrm{He}$ as a function of $\hbar \Omega$ at four different values of $\lambda$ $\left(\infty, 3,2,1.5 \mathrm{fm}^{-1}\right)$. The initial potential is the $500 \mathrm{MeV} \mathrm{N} \mathrm{NO}^{3} \mathrm{NN}$-only potential from Ref. [13]. The legend from Fig. 1 applies here.
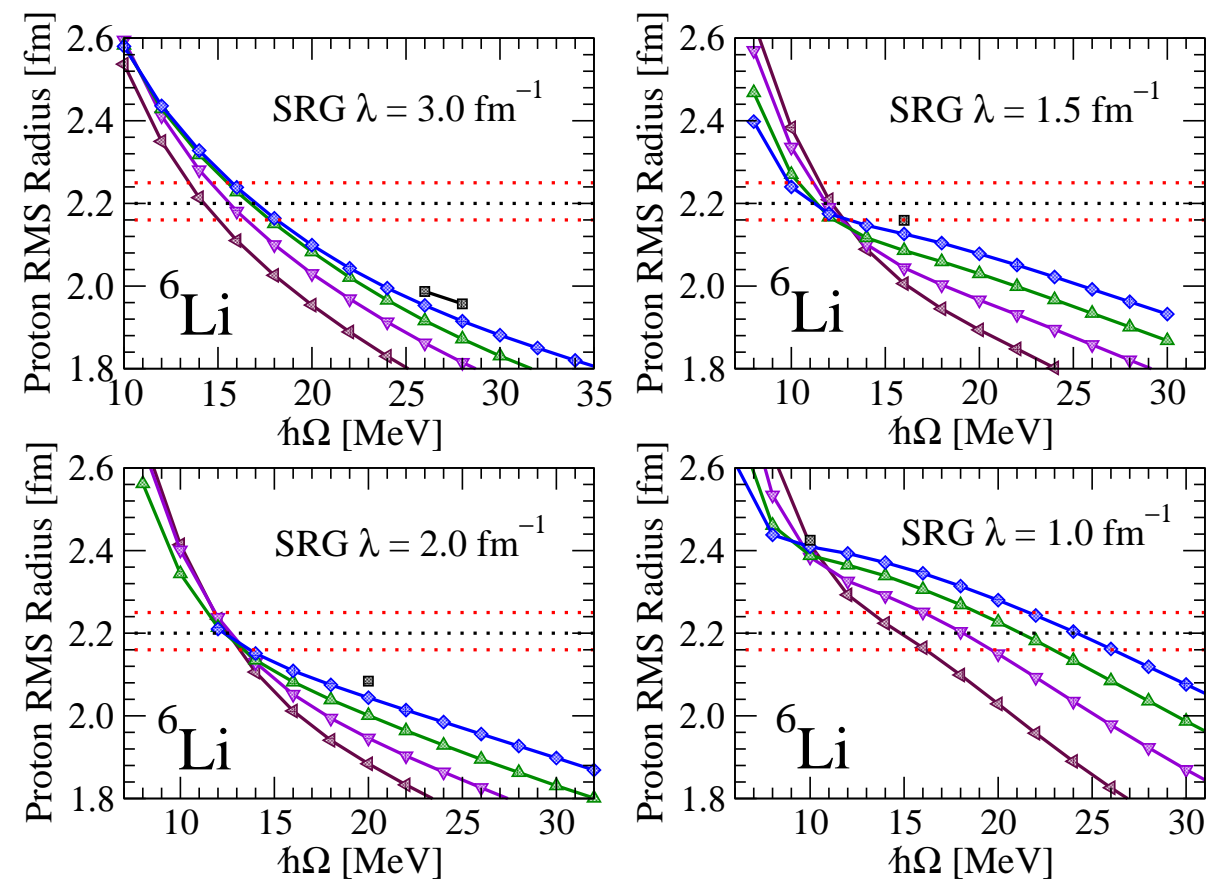

Fig. 24. Point proton radii of ${ }^{6} \mathrm{Li}$ as a function of $\hbar \Omega$ at four different values of $\lambda(3$, $\left.2,1.5,1 \mathrm{fm}^{-1}\right)$. The initial potential is the $500 \mathrm{MeV} \mathrm{N}{ }^{3} \mathrm{LO}$ NN-only potential from Ref. [13. The three dotted lines are radii from NCSM Lee-Suzuki calculations using the bare $\mathrm{N}^{3} \mathrm{LO}$ potential in larger spaces [18. The middle (black) line is without $3 \mathrm{~N}$ interactions and the others are with two different $3 \mathrm{~N}$ force fits [18. The legend from Fig. 5 applies here. 


\section{Summary}

We have performed NCSM calculations that retain the variational principle using RG-evolved potentials in momentum representation, focusing on the SRG approach that has recently been applied to nuclear structure physics. While we have not performed an exhaustive test of alternative potentials, we have found no sign of any special dependence on the initial potential. The convergence of ground-state energies improves rapidly with decreasing $\lambda$ and with no unnatural contributions from omitted three-body forces for these light nuclei. Furthermore, all methods of evolution to low momentum give similar convergence improvements, with smoother behavior associated with smoother cutoffs.

With these calculations we have set the stage for including $3 \mathrm{~N}$ interactions [23]. Just as varying $\lambda$ in Figs. 14 to 16 shows the scale of omitted many-body contributions, similar figures when $3 \mathrm{~N}$ is included will quantify the net impact of higher-order interactions. In the short term, three-body forces will be included in the form of $\mathrm{N}^{2} \mathrm{LO}$ chiral $3 \mathrm{~N}$ interactions, with the parameters fitted (see Ref. [8] for motivation). The development of evolved 3N potentials is proceeding in parallel and should be available in the near future. Once we have consistent $3 \mathrm{~N}$ interactions, we will be able to establish lower bounds to theoretical error bars for light nuclei by calculating with different $\mathrm{N}^{3} \mathrm{LO}$ cutoffs and assess the impact of uncertainties in the input chiral $3 \mathrm{~N}$ interactions.

\section{Acknowledgements}

We thank E. Anderson, E. Jurgenson, A. Nogga, S. Ramanan and the referee for useful comments. This work was supported in part by the National Science Foundation under Grant Nos. PHY-0354916 and PHY-0653312, the Department of Energy under Grant No. DE-FG02-87ER40371, the UNEDF SciDAC Collaboration under DOE Grant DE-FC02-07ER41457, and the Natural Sciences and Engineering Research Council of Canada (NSERC). TRIUMF receives federal funding via a contribution agreement through the National Research Council of Canada.

\section{References}

[1] S.K. Bogner, A. Schwenk, T.T.S. Kuo and G.E. Brown, nucl-th/0111042,

[2] S.K. Bogner, T.T.S. Kuo, A. Schwenk, D.R. Entem and R. Machleidt, Phys. Lett. B 576 (2003) 265. 
[3] S.K. Bogner, T.T.S. Kuo and A. Schwenk, Phys. Rept. 386 (2003) 1.

[4] S.K. Bogner, A. Schwenk, R.J. Furnstahl and A. Nogga, Nucl. Phys. A763 (2005) 59.

[5] S.K. Bogner, R.J. Furnstahl, S. Ramanan and A. Schwenk, Nucl. Phys. A784 (2007) 79 .

[6] S.K. Bogner, R.J. Furnstahl and R.J. Perry, Phys. Rev. C 75 (2007) 061001.

[7] H. Hergert and R. Roth, Phys. Rev. C 75 (2007) 051001.

[8] A. Nogga, S.K. Bogner and A. Schwenk, Phys. Rev. C 70 (2004) 061002(R).

[9] S.K. Bogner, R.J. Furnstahl, S. Ramanan and A. Schwenk, Nucl. Phys. A773 (2006) 203.

[10] S.K. Bogner and R.J. Furnstahl, Phys. Lett. B 632 (2006) 501; Phys. Lett. B 639 (2006) 237.

[11] G. Hagen, T. Papenbrock, D.J. Dean, A. Schwenk, A. Nogga, M. Wloch and P. Piecuch, Phys. Rev. C 76 (2007) 034302.

[12] G. Hagen, D.J. Dean, M. Hjorth-Jensen, T. Papenbrock and A. Schwenk, Phys. Rev. C 76 (2007) 044305.

[13] D.R. Entem and R. Machleidt, Phys. Rev. C 68 (2003) 041001(R).

[14] E. Epelbaum, W. Glöckle and U.G. Meißner, Nucl. Phys. A747 (2005) 362.

[15] P. Navrátil, J.P. Vary and B.R. Barrett, Phys. Rev. Lett. 84 (2000) 5728; Phys. Rev. C 62 (2000) 054311.

[16] P. Navrátil and W.E. Ormand, Phys. Rev. Lett. 88 (2002) 152502.

[17] P. Navrátil and W.E. Ormand, Phys. Rev. C 68 (2003) 034305.

[18] A. Nogga, P. Navrátil, B.R. Barrett and J.P. Vary, Phys. Rev. C 73 (2006) 064002 .

[19] P. Navrátil, V.G. Gueorguiev, J.P. Vary, W.E. Ormand and A. Nogga, Phys. Rev. Lett. 99 (2007) 042501.

[20] A.M. Shirokov, A.I. Mazur, S.A. Zaytsev, J.P. Vary and T.A. Weber, Phys. Rev. C 70 (2004) 044005.

[21] A.M. Shirokov, J.P. Vary, A.I. Mazur, S.A. Zaytsev and T.A. Weber, Phys. Lett. B 621 (2005) 96.

[22] A.M. Shirokov, J.P. Vary, A.I. Mazur and T.A. Weber, Phys. Lett. B 644 (2007) 33.

[23] S.K. Bogner, R.J. Furnstahl and R.J. Perry, arXiv:0708.1602,

[24] S.K. Bogner, R.J. Furnstahl, R.J. Perry and A. Schwenk, Phys. Lett. B 649 (2007) 488. 
[25] S.D. Glazek and K.G. Wilson, Phys. Rev. D 48 (1993) 5863; Phys. Rev. D 49 (1994) 4214.

[26] F. Wegner, Ann. Phys. (Leipzig) 3 (1994) 77.

[27] S. Szpigel and R.J. Perry, in Quantum Field Theory, A 20th Century Profile, Ed. A.N. Mitra, Hindustan Publishing Com., New Delhi, 2000, arXiv:hep-ph/0009071.

[28] See http://www.physics.ohio-state.edu/ ntg/srg/ for documentary examples.

[29] R.B. Wiringa, V.G.J. Stoks and R. Schiavilla, Phys. Rev. C 51 (1995) 38.

[30] I. Stetcu, B.R. Barrett, P. Navratil and J.P. Vary, Phys. Rev. C 71 (2005) 044325.

[31] J.P. Vary, The Many-Fermion Dynamics Shell-Model Code, Iowa State University (1992) (unpublished); J.P. Vary and D.C. Zheng, ibid., (1994) (unpublished).

[32] P. Navrátil, G.P. Kamuntavicius and B.R. Barrett, Phys. Rev. C 61 (2000) 044001.

[33] P. Maris, J.P. Vary and A. Shirokov, in preparation.

[34] E.D. Jurgenson, S.K. Bogner, R.J. Furnstahl, and R.J. Perry, arXiv:0711.4252,

[35] R. Roth, H. Hergert, P. Papakonstantinou, T. Neff and H. Feldmeier, Phys. Rev. C 72 (2005) 034002.

[36] P. Navrátil and E. Caurier, Phys. Rev. C 69 (2004) 014311.

[37] A. Nogga, H. Kamada and W. Glöckle, Phys. Rev. Lett. 85 (2000) 944.

[38] G. Hagen, D.J. Dean and A. Schwenk, in preparation.

[39] I. Stetcu, B.R. Barrett, P. Navrátil and J.P. Vary, Phys. Rev. C 71 (2005) 044325; Phys. Rev. C 73 (2006) 037307. 